This item was submitted to Loughborough's Research Repository by the author.

Items in Figshare are protected by copyright, with all rights reserved, unless otherwise indicated.

\title{
Direct estimation of marginal characteristics of nonparametric production frontiers in the presence of undesirable outputs
}

PLEASE CITE THE PUBLISHED VERSION

https://doi.org/10.1016/j.ejor.2019.05.024

PUBLISHER

(C) Elsevier

VERSION

AM (Accepted Manuscript)

\section{PUBLISHER STATEMENT}

This paper was accepted for publication in the journal European Journal of Operational Research and the definitive published version is available at https://doi.org/10.1016/j.ejor.2019.05.024

LICENCE

CC BY-NC-ND 4.0

\section{REPOSITORY RECORD}

Podinovski, Victor. 2019. "Direct Estimation of Marginal Characteristics of Nonparametric Production Frontiers in the Presence of Undesirable Outputs". Loughborough University. https://hdl.handle.net/2134/37813. 


\title{
Direct estimation of marginal characteristics of nonparametric production frontiers in the presence of undesirable outputs
}

\author{
Victor V. Podinovski ${ }^{\mathrm{a}}$ \\ ${ }^{a}$ School of Business and Economics, Loughborough University, Loughborough LE11 3TU, UK
}

\begin{abstract}
There is extensive literature on the estimation of marginal characteristics of nonparametric production frontiers, including various marginal rates and elasticity measures. It has recently been shown that all such characteristics can be evaluated by a unifying linear programming approach applicable to any polyhedral production technology. In this paper we show how this approach can be applied to polyhedral technologies incorporating undesirable outputs. In particular, we derive a linear programming method for the direct assessment of the marginal rate of transformation between a bad and a good output often used for the estimation of the unobserved price of the bad output. In contrast with the existing methods based on a conventionally specified directional distance function, the new approach does not require the assessment of two shadow prices of the good and bad outputs. It also correctly estimates one-sided marginal rates in all cases in which the shadow prices on nonsmooth production frontiers are not unique.
\end{abstract}

Keywords: Data envelopment analysis, Directional distance function, Undesirable output, Marginal rate, Shadow price

\section{Introduction}

Marginal characteristics of production frontiers such as marginal rates of substitution and transformation between inputs and outputs, and various elasticity measures, play an important role in applications of productivity and efficiency analysis. The literature on this subject is extensive, both for the parametric and nonparametric specifications of the production technology. Balk, Färe, and Karagiannis (2015) provide a review of approaches for the evaluation of scale elasticity in the neoclassical parametric setting. Reviews of analogous approaches in the nonparametric framework of data envelopment analysis (DEA) are found in Banker, Cooper, Seiford, and Zhu (2011), and Sahoo and Tone (2015). Asmild, Paradi and Reese (2006) provide a review of the evaluation methods of various marginal rates in the framework of DEA. Zhou, Zhou and Fan (2014) survey the evaluation of marginal rates in models with undesirable outputs using both the parametric and nonparametric methodologies.

Recently, Podinovski, Chambers, Atici, and Deineko (2016) developed a unifying linear programming approach to the evaluation of such characteristics in a very large class of polyhedral production technologies. A non-exhaustive list of polyhedral technologies includes the standard constant and variable returns-to-scale (CRS and VRS) technologies of Charnes, Cooper, and Rhodes (1978) and Banker, Charnes, and Cooper (1984), their extensions with

Email address: v.podinovski@lboro.ac.uk (Victor V. Podinovski) 
production trade-offs and weight restrictions (Podinovski, 2004b; Podinovski \& BouzdineChameeva, 2013), technologies with weakly disposable inputs and outputs (Mehdiloo \& Podinovski, 2019; Mehdiloozad \& Podinovski, 2018), technologies with multiple component processes (Cherchye, De Rock, Dierynck, Roodhooft, \& Sabbe, 2013; Podinovski, Olesen, \& Sarrico, 2018), and various network technologies (Kao, 2014). Podinovski et al. (2016) prove that the evaluation of different marginal characteristics in any polyhedral technology can be performed by solving essentially the same linear programs. Such programs take on a specific form for different marginal characteristics of interest and the assumed production technology.

In this paper, we show how the above unifying approach can be applied to technologies incorporating undesirable (bad) outputs. Dakpo, Jeanneaux, and Latruffe (2016) and Pham and Zelenyuk (2019) provide a critical review of models of technologies with undesirable outputs, many of which are polyhedral technologies. A new two-stage network polyhedral technology with undesirable outputs in the banking sector has recently been developed by Fukuyama and Matousek (2018).

Note that, in the standard setting without bad outputs, the production frontier is defined by the maximization of all outputs and the minimization of all inputs. In contrast, the production frontier of a technology with bad outputs is defined by the maximization of only good outputs and the minimization of the inputs and bad outputs. This difference requires a different specification of the directional distance function of Chambers, Chung, and Färe $(1996,1998)$ used to represent the technology. This further requires a different specification of the linear programs used in the approach of Podinovski et al. (2016) that relies on a correct representation of the production frontier by the directional distance function.

In this paper, we address the above differences and clarify the application of the unifying approach of Podinovski et al. (2016) to technologies with undesirable outputs. To be specific, we consider the technology of Kuosmanen (2005) which assumes joint weak disposability of the bad and good outputs, and state all our results in terms of this technology. We further show that any other polyhedral technology with bad outputs would require a similar treatment.

To give this paper a more practical focus, we consider a number of specific marginal rates and elasticity measures that have been extensively studied in the literature. In particular, the unobserved prices of bad outputs have traditionally been assessed using the ratio of the shadow prices of the bad and good outputs evaluated for an appropriately specified directional distance function (Färe, Grosskopf, Noh, \& Weber, 2005). A known problem with this approach arises from the fact that such shadow prices are usually not unique (Førsund, 2018). The estimation of the minimal and maximal ratios of such shadow prices, which are needed in order to obtain the range of the unobserved prices of the bad output, requires solving two computationally unattractive nonlinear programs.

In contrast, we show that the unobserved prices of bad outputs can instead be evaluated directly, by using the partial derivative of the good output with respect to the bad output calculated by the appropriately specified linear programs of Podinovski et al. (2016). This approach has two advantages. Firstly, it requires assessing only one shadow price which is theoretically equal to the negated ratio of the two shadow prices in the conventional approach. Secondly, and more importantly, the estimation of the minimal and maximal values of the ratio of the two shadow prices in the conventional approach which leads to two nonlinear programs is, in the suggested direct approach, a simple task of assessing the minimal and maximal values of a single shadow price. The latter task is straightforward and requires solving only linear programs.

We proceed as follows. In Section 2, we briefly introduce the technology of Kuosmanen 
(2005) with bad outputs and show the difference in the definition of strong efficiency in this technology with the standard case in which the bad outputs are not specified. In Section 3, we state the main result that allows us to evaluate the one-sided directional derivatives of the directional distance function. In Section 4, we use this main result to obtain linear programs for the direct evaluation of various marginal rates. In Section 5,we show how these results could be used for the direct estimation of the unknown price of an undesirable output. In Sections 6 and 7, we consider the general approach to the evaluation of elasticity measures and its special cases, respectively. In Section 8, we use numerical examples to illustrate the use of the developed linear programming approach. In Section 9, we consider an extended example which we use to compare the suggested direct approach to the estimation of marginal rates with the conventional approach based on the use of shadow prices in the directional distance function model. Section 10 contains concluding remarks. In Appendix A and Appendix B, we provide additional results and examples that complement our main discussion. All mathematical proofs are given in Appendix C.

\section{The technology of Kuosmanen (2005)}

The technology developed by Kuosmanen (2005) and further explored in Kuosmanen and Podinovski (2009), Kuosmanen and Kazemi Matin (2011) and Podinovski and Kuosmanen (2011), is one of several ways of modelling joint production of good and undesirable outputs developed in the literature. Below we denote this technology $\mathcal{T}_{\mathrm{K}}$. The undesirable outputs are assumed to be weakly disposable together with the good outputs. The inputs and good outputs are assumed to be strongly (freely) disposable.

Let $X \in \mathbb{R}_{+}^{m}$ be the vector of inputs, and let $V \in \mathbb{R}_{+}^{s}$ and $W \in \mathbb{R}_{+}^{p}$ be the vectors of good and bad outputs, respectively. Denote observed production units $\left(X_{(j)}, V_{(j)}, W_{(j)}\right)$, $j=1, \ldots, n$.

As shown by Kuosmanen and Podinovski (2009), technology $\mathcal{T}_{\mathrm{K}}$ can be defined as the smallest technology (in the sense of the minimum extrapolation principle of Banker et al., 1984) that contains all observed units and satisfies the following axioms:

A1 (Strong disposability of inputs and good outputs) If $(X, V, W) \in \mathcal{T}_{\mathrm{K}}$, then $\left(X^{\prime}, V^{\prime}, W\right) \in$ $\mathcal{T}_{\mathrm{K}}$, for all $X^{\prime} \geq X$ and $0 \leq V^{\prime} \leq V$.

A1 (Weak disposability of good and undesirable outputs) If $(X, V, W) \in \mathcal{T}_{\mathrm{K}}$, then $(X, \theta V, \theta W) \in$ $\mathcal{T}_{\mathrm{K}}$, for all $\theta \in[0,1]$.

A3 (Convexity) $\mathcal{T}_{\mathrm{K}}$ is a convex set.

To obtain an explicit statement of technology $\mathcal{T}_{\mathrm{K}}$, let $\bar{X}, \bar{V}$ and $\bar{W}$ be the matrices whose columns are the vectors $X_{(j)}, V_{(j)}$ and $W_{(j)}, j=1, \ldots, n$, of the observed units, respectively.

Following Kuosmanen (2005), technology $\mathcal{T}_{\mathrm{K}}$ contains all nonnegative units $(X, V, W)$ for which there exist vectors $\lambda, \mu \in \mathbb{R}^{n}$ and slack vectors $S_{X} \in \mathbb{R}^{m}$ and $S_{V} \in \mathbb{R}^{s}$ such that the following conditions are true:

$$
\begin{aligned}
& \bar{X} \lambda+\bar{X} \mu+S_{X}=X, \\
& \bar{V} \lambda-S_{V}=V, \\
& \bar{W} \lambda=W, \\
& 1^{\top} \lambda+1^{\top} \mu=1, \\
& \lambda, \mu, S_{X}, S_{V} \geq 0 .
\end{aligned}
$$


Technology $\mathcal{T}_{\mathrm{K}}$ is an example of a very large class of polyhedral technologies introduced by Podinovski et al. (2016). ${ }^{1}$ As shown in the latter paper, the evaluation of various marginal characteristics in all polyhedral technologies can be performed by solving the same linear programs stated in a unifying general form that need to be correctly specified for the technology and the marginal characteristic of interest.

Such specification requires a correct representation of the production frontier of the technology by the directional distance function of Chambers et al. (1998), depending on the marginal characteristic of interest and the definition of efficient production which is different in technologies with and without bad outputs. Indeed, while the efficient frontier of a technology with only good outputs is defined by the maximization of all outputs and minimization of all inputs (in the Pareto sense), the efficient frontier of a technology with bad outputs is defined by the maximization of only the good outputs and minimization of the inputs and bad outputs. For example, for technology $\mathcal{T}_{\mathrm{K}}$, we have the following definition.

Definition 1. The unit $(X, V, W) \in \mathcal{T}_{\mathrm{K}}$ is strongly efficient if there does not exist a unit $\left(X^{\prime}, V^{\prime}, W^{\prime}\right) \in \mathcal{T}_{\mathrm{K}}$ such that $X^{\prime} \leq X, V^{\prime} \geq V$ and $W^{\prime} \leq W$, and $\left(X^{\prime}, V^{\prime}, W^{\prime}\right) \neq(X, V, W)$.

As an illustration, consider technology $\mathcal{T}_{\mathrm{K}}$ generated by observed units $A, B, C$ and $D$, shown in two output dimensions $V$ and $W$ in Fig. 1. The input levels $X$ of all observed units are assumed to be the same and are not shown. The vertical lines drawn downwards from the observed units represent strong disposability of the good output $V$. The lines from the observed units towards the origin represent simultaneous proportional reduction of both outputs and correspond to the assumption of their joint weak disposability.

According to Definition 1, the strongly efficient frontier of technology $\mathcal{T}_{\mathrm{K}}$ in this example is the broken line $O A B$. In contrast, if both outputs were assumed to be good outputs, the strongly efficient frontier of the same technology (or of the larger VRS technology based on the same observed units) would be $B C D .^{2}$

\section{Theoretical foundations}

Below we show how the main theoretical results developed for a general polyhedral technology by Podinovski et al. (2016) are applied in the case of technology $\mathcal{T}_{\mathrm{K}}$, and obtain their more specific statements. We use these results in subsequent sections for the evaluation of various marginal rates and elasticity measures.

\subsection{Directional response function}

Following the framework used by Podinovski and Førsund (2010) and its extension in Podinovski et al. (2016), we consider the partition of all inputs and all (good and bad) outputs into the three mutually disjoint sets $A, B$ and $C$, where the sets $A$ and $B$ are not empty and the set $C$ may be empty.

Let a unit $\left(X_{o}, V_{o}, W_{o}\right)$ be located on the production frontier (as formally defined in Assumption 1 below). We are interested in the evaluation of the marginal changes of the

\footnotetext{
${ }^{1}$ This class is stated in terms of the input and output vectors $X$ and $Y$ whose components (individual inputs and outputs) may or may not satisfy the assumption of strong disposability. The classification of the measures that are not strongly disposable into inputs and outputs is unimportant as both lead to equivalent linear programs for the evaluation of marginal characteristics of the production frontiers.

${ }^{2}$ For the strongly disposable VRS technology in which both $V$ and $W$ are assumed to be good outputs, we add a horizontal line to the left of point $B$ (not shown) and the area below it. This horizontal line and the vertical line descending from $D$ include weakly efficient units of the VRS technology.
} 


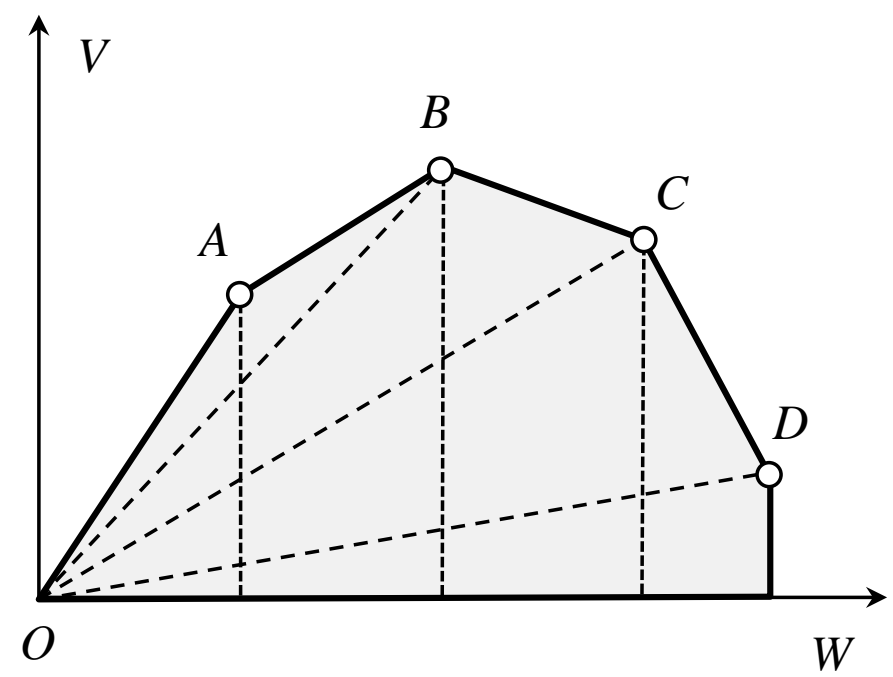

Figure 1: The section of technology $\mathcal{T}_{\mathrm{K}}$ in dimensions $W$ and $V$, for a fixed $X$.

input and output measures of this unit in the set $B$ with respect to marginal changes of the measures in the set $A$, while keeping the measures in the set $C$ constant. These marginal changes are taken in some pre-specified directions (which allow us to consider a variety of different scenarios), assuming that the resulting unit remains on the production frontier.

To state this task formally, and taking into account the partition of all inputs and outputs into the sets $A, B$ and $C$, we represent any unit in technology $\mathcal{T}_{\mathrm{K}}$ as follows:

$$
\left(X_{o}, V_{o}, W_{o}\right)=\left(X_{o}^{A}, X_{o}^{B}, X_{o}^{C}, V_{o}^{A}, V_{o}^{B}, V_{o}^{C}, W_{o}^{A}, W_{o}^{B}, W_{o}^{C}\right) .
$$

In the above statement, the superscripts identify the subvectors of the inputs and good and bad outputs included in the corresponding sets $A, B$ and $C$.

To keep the setting as general as possible, let the vectors $\Delta^{A}=\left(\Delta_{X}^{A}, \Delta_{V}^{A}, \Delta_{W}^{A}\right)$ and $\Delta^{B}=\left(\Delta_{X}^{B}, \Delta_{V}^{B}, \Delta_{W}^{B}\right)$ describe the directions of marginal perturbations of the measures in the sets $A$ and $B$, respectively. For example, the subvector $\Delta_{X}^{A}$ describes marginal perturbations of the vector $X_{o}^{A}$. This vector is undefined and omitted if the set $A$ does not include inputs.

Following Podinovski et al. (2016), define the directional response function whose argument is the amount $\gamma$ of perturbation $\Delta^{A}$ applied to the measures in the set $A$. The value of this function is equal to the maximum amount $\varphi$ of perturbation $\Delta^{B}$ of the measures in the set $B$ that keeps the resulting unit in technology $\mathcal{T}_{\mathrm{K}}$, i.e., on its boundary.

Definition 2. For the unit $\left(X_{o}, V_{o}, W_{o}\right) \in \mathcal{T}_{\mathrm{K}}$ and predefined vectors $\Delta^{A}$ and $\Delta^{B}$, the directional response function $\bar{\varphi}(\gamma)$ is the optimal value of the following program in which $\gamma$ is a parameter:

$$
\begin{aligned}
\bar{\varphi}(\gamma)=\max \{ & \varphi \mid\left(X_{o}^{A}+\gamma \Delta_{X}^{A}, X_{o}^{B}+\varphi \Delta_{X}^{B}, X_{o}^{C},\right. \\
& V_{o}^{A}+\gamma \Delta_{V}^{A}, V_{o}^{B}+\varphi \Delta_{V}^{B}, V_{o}^{C}, \\
& \left.\left.W_{o}^{A}+\gamma \Delta_{W}^{A}, W_{o}^{B}+\varphi \Delta_{W}^{B}, W_{o}^{C}\right) \in \mathcal{T}_{\mathrm{K}}, \varphi \in \mathbb{R}\right\} .
\end{aligned}
$$

Taking into account the statement (1) of technology $\mathcal{T}_{\mathrm{K}}$, note that, for each value $\gamma$, program (2) is a linear program. The complete extended statement of this program is given in Appendix C. 
The notion of marginal rates and elasticity measures applies to the units on the production frontier. For the chosen direction of response $\Delta^{B}$, any unit $\left(X_{o}, V_{o}, W_{o}\right)$ on such frontier satisfies the following assumption.

Assumption 1. The unit $\left(X_{o}, V_{o}, W_{o}\right)$ is weakly efficient in the direction $\Delta^{B}$, i.e., $\bar{\varphi}(0)=0$.

It is clear that $\bar{\varphi}(\gamma)$ is the directional distance function of Chambers et al. (1998) defined by the direction $\Delta^{B}$, for all units obtained from $\left(X_{o}, V_{o}, W_{o}\right)$ by the parameterized perturbation $\gamma \Delta^{A}$. According to Assumption 1, the unit $\left(X_{o}, V_{o}, W_{o}\right)$ is located on the weakly efficient frontier defined by this directional distance function.

In the next sections we show that different marginal characteristics of the production frontier can be obtained as the one-sided derivatives $\bar{\varphi}_{+}^{\prime}(0)$ and $\bar{\varphi}_{-}^{\prime}(0)$ of the function $\bar{\varphi}(\gamma)$ defined by the appropriately specified directions $\Delta^{A}$ and $\Delta^{B}$.

For example, let the set $A$ contain a bad output $l^{\prime}$, the set $B$ contain a good output $r^{\prime}$, and the set $C$ contain all the other inputs and good and bad outputs. In this case, the vectors $\Delta^{A}=\Delta_{W}^{A} \in \mathbb{R}^{1}$ and $\Delta^{B}=\Delta_{V}^{B} \in \mathbb{R}^{1}$ each have a single component. Let both such components be equal to 1 , i.e., let $\left(\Delta_{W}^{A}\right)_{l^{\prime}}=1$ and $\left(\Delta_{V}^{B}\right)_{r^{\prime}}=1$. For this specification, if the bad output is increased by $\gamma$, i.e., from $\left(W_{o}\right)_{l^{\prime}}$ to $\left(W_{o}\right)_{l^{\prime}}+\gamma$, the corresponding maximum good output possible in technology $\mathcal{T}_{\mathrm{K}}$ (i.e., observed on the production frontier) increases from $\left(V_{o}\right)_{r^{\prime}}$ to $\left(V_{o}\right)_{r^{\prime}}+\bar{\varphi}(\gamma)$. Then the right-hand derivative $\bar{\varphi}_{+}^{\prime}(0)$ represents the right-hand marginal rate of transformation $\partial^{+} V_{r^{\prime}} / \partial W_{l^{\prime}}$ of the good output $V_{r^{\prime}}$ with respect to marginal changes of the bad input $W_{l^{\prime}}$, evaluated at the unit $\left(X_{o}, V_{o}, W_{o}\right)$ on the frontier. Similarly, the left-hand derivative $\bar{\varphi}_{-}^{\prime}(0)$ represents the left-hand marginal rate of transformation $\partial^{-} V_{r^{\prime}} / \partial W_{l^{\prime}}$ evaluated at the same unit. In Section 5 we show how these two one-sided derivatives can be used for pricing bad outputs.

As another example demonstrating the flexibility of the described approach based on the use of the function $\bar{\varphi}(\gamma)$, let the set $A$ include all inputs, the set $B$ include all outputs and $C$ include all bad outputs. As shown in Section 6, if we define the perturbation vectors $\Delta^{A}=\Delta_{X}^{A}=X_{o}^{A}$ and $\Delta^{B}=\Delta_{Y}^{B}=Y_{o}^{B}$, the one-sided derivatives $\bar{\varphi}_{+}^{\prime}(0)$ and $\bar{\varphi}_{-}^{\prime}(0)$ are equal to the right-hand and left-hand partial scale elasticity evaluated at the unit $\left(X_{o}, V_{o}, W_{o}\right)$, under the scenario in which we keep the bad output constant.

\subsection{Evaluation of the one-sided derivatives of the directional response function}

Methods of evaluation of the one-sided derivatives $\bar{\varphi}_{+}^{\prime}(0)$ and $\bar{\varphi}_{-}^{\prime}(0)$ in different technologies were developed in the literature in two different but equivalent theoretical frameworks. Chambers and Färe (2008) state this approach for the VRS technology in the language of directional derivatives of a directional distance function using general results of nondifferentiable convex optimization. Podinovski, Førsund, and Krivinozhko (2009) and Podinovski and Førsund (2010) develop a more specific and operational approach based on sensitivity analysis in linear programming. Podinovski et al. (2016) extend the latter approach to any polyhedral technology, a particular example of which is technology $\mathcal{T}_{\mathrm{K}}$. Below we follow this last approach and show that its application to technology $\mathcal{T}_{\mathrm{K}}$ gives us a method for direct estimation of a variety of marginal characteristics.

It turns out that, regardless of the choice of vectors $\Delta^{A}$ and $\Delta^{B}$ and the marginal characteristics that they define, the evaluation of the one-sided derivatives $\bar{\varphi}_{+}^{\prime}(0)$ and $\bar{\varphi}_{-}^{\prime}(0)$ requires solving very similar linear programs that are variations of the same general linear program. Namely, the right-hand derivative $\bar{\varphi}_{+}^{\prime}(0)$ is evaluated as the minimum of the scalar product of the vector $\left(\Delta_{X}^{A},-\Delta_{V}^{A}, \Delta_{W}^{A}\right)$ and the vector of the corresponding dual variables taken over the set of optimal solutions to the dual of program (2) stated for $\gamma=0$. Similarly, 
the left-hand derivative $\bar{\varphi}_{-}^{\prime}(0)$ is evaluated as the maximum of the same scalar product taken over the same set of dual optimal solutions.

The next theorem establishes this result formally. It is a variant of the main theorem proved in Podinovski et al. (2016) and restated for technology $\mathcal{T}_{\mathrm{K}}$. It is stated in terms of vectors $v=\left(v^{A}, v^{B}, v^{C}\right), u=\left(u^{A}, u^{B}, u^{C}\right)$ and $z=\left(z^{A}, z^{B}, z^{C}\right)$ interpretable at optimality as the shadow prices, or weights, of the inputs and good and bad outputs, respectively. The superscripts identify the subvectors of the vectors $v, u$ and $z$ corresponding to the sets $A, B$ and $C$. As shown in Appendix $\mathrm{C}$, these vectors are dual to the input and output constraints of the linear program (2) stated with $\gamma=0$. The sign-free variable $\omega$ is dual to the normalizing equality (1d).

For a rigorous statement of the next theorem, we also need the following definition. It reflects the fact that program (2) is generally correctly defined (is feasible and has a finite optimal value) not for all $\gamma$ but only for a certain range denoted $\Gamma$, and the interpretation of the one-sided derivatives $\bar{\varphi}_{+}^{\prime}(0)$ and $\bar{\varphi}_{-}^{\prime}(0)$ depends on whether $\gamma=0$ is an interior or extreme point of $\Gamma$.

Formally, let $\Gamma$ be the domain of the function $\bar{\varphi}(\gamma)$. By definition, it includes all values $\gamma$ for which linear program (2) is feasible and has a finite optimal value. According to Assumption 1, we have $\gamma=0 \in \Gamma$. Therefore, the domain $\Gamma$ is not an empty set. Based on known results of sensitivity analysis, Podinovski et al. (2016) shows that $\Gamma$ is a closed interval. Furthermore, $\bar{\varphi}(\gamma)$ is a continuous, concave and piecewise linear function on $\Gamma$. Therefore, if $\gamma=0$ is not the right extreme point of the interval $\Gamma$, then $\bar{\varphi}(\gamma)$ is a linear function in some right neighbourhood of the point $\gamma=0$. In this case the right-hand derivative $\bar{\varphi}_{+}^{\prime}(0)$ exists and is finite. Similarly, if $\gamma=0$ is not the left extreme point of the interval $\Gamma$, then $\bar{\varphi}(\gamma)$ is a linear function in some left neighbourhood of the point $\gamma=0$. This implies that the left-hand derivative $\bar{\varphi}_{-}^{\prime}(0)$ exists and is finite.

We also require the following assumption, which should be unproblematic in most practical applications. It guarantees that marginal perturbations of the unit $\left(X_{o}, V_{o}, W_{o}\right)$ in the directions defined by vectors $\Delta^{A}$ and $\Delta^{B}$ do not result in negative values of the good outputs in the perturbed unit. ${ }^{3}$

Assumption 2. All good outputs of the unit $\left(X_{o}, V_{o}, W_{o}\right)$ included in the sets $A$ and $B$ are strictly positive, i.e., $V_{o r}>0$, for all $r \in A \cup B$.

In Appendix A, we show that Assumption 2 can be significantly relaxed. We demonstrate by examples that, without Assumption 2 (or its relaxed analogue), the next result is generally no longer valid. We also obtain a more general variant of the next theorem which does not require this assumption and applies to any unit $\left(X_{o}, V_{o}, W_{o}\right) \in \mathcal{T}_{\mathrm{K}}$.

Theorem 1. Let $\left(X_{o}, V_{o}, W_{o}\right) \in \mathcal{T}_{\mathrm{K}}$, and let Assumption 2 be satisfied.

1. If Assumption 1 is satisfied then the following four statements are true:

(i) If $\gamma=0$ is not the right extreme point of domain $\Gamma$, then the right-hand derivative

\footnotetext{
${ }^{3}$ In the proof of a more general Theorem 3 given in Appendix C, we show that the nonnegativity of the perturbed inputs and bad outputs follows from the constraints of program (3) and does not require any additional assumptions.
} 
$\bar{\varphi}_{+}^{\prime}(0)$ exists, is finite and is equal to the optimal value of the linear program

$$
\begin{aligned}
& \bar{\varphi}_{+}^{\prime}(0)=\min \left(\Delta_{X}^{A}\right)^{\top} v^{A}-\left(\Delta_{V}^{A}\right)^{\top} u^{A}+\left(\Delta_{W}^{A}\right)^{\top} z^{A} \\
& \text { s.t. }-\left(\Delta_{X}^{B}\right)^{\top} v^{B}+\left(\Delta_{V}^{B}\right)^{\top} u^{B}-\left(\Delta_{W}^{B}\right)^{\top} z^{B}=1, \\
& X_{o}^{\top} v-V_{o}^{\top} u+W_{o}^{\top} z+\omega=0, \\
& \bar{X}^{\top} v-\bar{V}^{\top} u+\bar{W} z+1 \omega \geq 0, \\
& \bar{X}^{\top} v+1 \omega \geq 0, \\
& u, v \geq 0, z, \omega \text { sign free. }
\end{aligned}
$$

(ii) If $\gamma=0$ is the right extreme point of $\Gamma$, then the optimal value of program (3) is unbounded.

(iii) If $\gamma=0$ is not the left extreme point of $\Gamma$, then the left-hand derivative $\bar{\varphi}_{-}^{\prime}(0)$ exists, is finite and is equal to the optimal value of the linear program

$$
\begin{aligned}
& \bar{\varphi}_{-}^{\prime}(0)=\max \left(\Delta_{X}^{A}\right)^{\top} v^{A}-\left(\Delta_{V}^{A}\right)^{\top} u^{A}+\left(\Delta_{W}^{A}\right)^{\top} z^{A} \\
& \text { s.t. }(3 \mathrm{~b})-(3 \mathrm{f}) .
\end{aligned}
$$

(iv) If $\gamma=0$ is the left extreme point of $\Gamma$, then the optimal value of program (4) is unbounded.

2. If Assumption 1 is not satisfied, then programs (3) and (4) are infeasible.

The proof of Theorem 1 and the other results are given in Appendix C.

It is straightforward to verify (see the proof of Theorem 3 in Appendix $\mathrm{C}$ ) that the constraints $(3 \mathrm{~b})$ and $(3 \mathrm{~d})-(3 \mathrm{f})$ are the constraints of the dual to the linear program (2) stated for $\gamma=0$. The equality (3c) states that the objective function of this dual is equal to 0 , which, according to Assumption 1, is the optimal value of program (2) for $\gamma=0$. This means that the feasible region of programs (3) and (4) is the set of optimal solutions $(u, v, z, \omega)$ of the dual to linear program (2) stated with $\gamma=0$. The objective function in program (3) minimizes the scalar product of the vector $\left(\Delta_{X}^{A},-\Delta_{V}^{A}, \Delta_{W}^{A}\right)$ and the combined vector of $\left(u^{A}, v^{A}, z^{A}\right)$ over this feasible set. Similarly, program (4) maximizes this scalar product over the same set.

\subsection{Interpretation of optimal solutions}

To illustrate the use of Theorem 1 , consider any unit $\left(X_{o}, V_{o}, W_{o}\right) \in \mathcal{T}_{\mathrm{K}}$ that satisfies Assumption 2. To be specific, consider the evaluation of the right-hand derivative $\bar{\varphi}_{+}^{\prime}(0)$ by solving program (3). (The evaluation of the left-hand derivative $\bar{\varphi}_{-}^{\prime}(0)$ by solving program (4) is similar.) Three computational outcomes are theoretically possible.

Case 1. Suppose that program (3) has a finite optimal solution. Then the unit $\left(X_{o}, V_{o}, W_{o}\right)$ satisfies Assumption 1 as otherwise, by statement 2 of Theorem 1, this program would be infeasible. Furthermore, $\gamma=0$ is not the right extreme point of the domain $\Gamma$ as otherwise, by statement 1(ii), the optimal value of program (3) would be unbounded. In words, the unit $\left(X_{o}, V_{o}, W_{o}\right)$ is located on the production frontier of technology $\mathcal{T}_{\mathrm{K}}$ and it is possible to change its input and output measures in the direction $\Delta^{A}$ while staying in the technology. Therefore, the unit $\left(X_{o}, V_{o}, W_{o}\right)$ satisfies the conditions of statement 1(i) of Theorem 1 and $\bar{\varphi}_{+}^{\prime}(0)$ is equal to the optimal value of program (3).

Case 2. Suppose that program (3) is feasible but has an unbounded optimal value. Then, similar to Case 1, the unit $\left(X_{o}, V_{o}, W_{o}\right)$ satisfies Assumption 1 . However, $\gamma=0$ is the 
right extreme point of the interval $\Gamma$ as otherwise, by statement $1(\mathrm{i})$, the optimal value of program (3) would be finite. This means that any marginal movement in the direction $\Delta^{A}$ leads outside technology $\mathcal{T}_{\mathrm{K}}$. For this reason, the right-hand derivative $\bar{\varphi}_{+}^{\prime}(0)$ at the unit $\left(X_{o}, V_{o}, W_{o}\right)$ is undefined.

Case 3. Suppose that program (3) is infeasible. It is easy to see that, in this case, the unit $\left(X_{o}, V_{o}, W_{o}\right)$ does not satisfy Assumption 1. Indeed, assuming the opposite means that, by the statements 1 (i) and 1(ii) of Theorem 1, program (3) is feasible and either has a finite or unbounded optimal value, which contradicts the conditions of Case 3. Because the unit $\left(X_{o}, V_{o}, W_{o}\right)$ does not satisfy Assumption 1, the right-hand derivative $\bar{\varphi}_{+}^{\prime}(0)$ is undefined.

It is worth noting that the described procedure does not require a preliminary testing of Assumption 1. By solving programs (3) and (4), we simultaneously identify those units that do not satisfy Assumption 1 by the infeasibility report of the linear programming solver.

We illustrate the described procedure and interpretations by numerical examples in Section 8 .

\subsection{Inefficient units}

Theorem 1 is valid under the condition that the unit $\left(X_{o}, V_{o}, W_{o}\right)$ satisfies Assumption 1. This means that this unit is located on the part of the production frontier that is weakly (radially) efficient in the direction $\Delta^{B}$. In this case, as shown in the next sections, the one-sided derivatives $\bar{\varphi}_{+}^{\prime}(0)$ and $\bar{\varphi}_{-}^{\prime}(0)$ are equal to particular one-sided marginal values evaluated at this unit and defined by the chosen directions $\Delta^{A}$ and $\Delta^{B}$.

In practical applications, it may also be interesting to consider the units that do not satisfy Assumption 1, in which case we have $\bar{\varphi}(0)>0$. Although marginal frontier characteristics are undefined for any such units, they are correctly defined at their projections on the production frontier. According to (2), the radial projection of the unit $\left(X_{o}, V_{o}, W_{o}\right)$ on the weakly efficient frontier taken in the direction of vector $\Delta^{B}$ is defined as

$$
\begin{aligned}
\left(X^{*}, V^{*}, W^{*}\right)= & \left(X_{o}^{A}, X_{o}^{B}+\bar{\varphi}(0) \Delta_{X}^{B}, X_{o}^{C},\right. \\
& V_{o}^{A}, V_{o}^{B}+\bar{\varphi}(0) \Delta_{V}^{B}, V_{o}^{C}, \\
& \left.W_{o}^{A}, W_{o}^{B}+\bar{\varphi}(0) \Delta_{W}^{B}, W_{o}^{C}\right) .
\end{aligned}
$$

Denote $\bar{\varphi}^{*}(\gamma)$ the directional response function for the projected unit, and let $\Gamma^{*}$ be its domain. The function $\bar{\varphi}^{*}(\gamma)$ is defined by $(2)$ where the unit $\left(X_{o}, V_{o}, W_{o}\right)$ is replaced by its projection $\left(X^{*}, V^{*}, W^{*}\right)$. It is clear that the projected unit $\left(X^{*}, V^{*}, W^{*}\right)$ satisfies Assumption 1, i.e., we have $\bar{\varphi}^{*}(0)=0$. Applying Theorem 1, the one-sided derivatives $\varphi_{+}^{* \prime}(0)=0$ and $\varphi^{* \prime}(0)=0$ can now be evaluated by solving programs (3) and (4), respectively, in which case the unit $\left(X_{o}, V_{o}, W_{o}\right)$ should be replaced by its projection $\left(X^{*}, V^{*}, W^{*}\right)$.

An alternative and possibly more practical way to evaluate the one-sided derivatives $\varphi_{+}^{* \prime}(0)$ and $\varphi_{-}^{* \prime}(0)$ at the projected unit is established by the following result.

Proposition 1. The domains $\Gamma^{*}$ and $\Gamma$ of the functions $\bar{\varphi}^{*}(\gamma)$ and $\bar{\varphi}(\gamma)$ coincide. The statements 1(i)-1(iv) of Theorem 1 remain valid for the projected unit $\left(X^{*}, V^{*}, W^{*}\right)$ with the following modification. ${ }^{4}$ The one-sided derivatives $\bar{\varphi}^{*_{+}^{\prime}}{ }_{(}(0)$ and $\bar{\varphi}^{*^{\prime}}{ }_{-}(0)$ evaluated at the projected unit $\left(X^{*}, V^{*}, W^{*}\right)$ are obtained by solving programs (3) and (4) in which the value 0 on the right-hand side of constraint $(3 \mathrm{c})$ is replaced by $\bar{\varphi}(0)$, i.e., by changing this constraint to

$$
X_{o}^{\top} v-V_{o}^{\top} u+W_{o}^{\top} z+\omega=\bar{\varphi}(0) \text {. }
$$

\footnotetext{
${ }^{4}$ Because the projected unit satisfies Assumption 1, the statement 2 of Theorem 1 does not apply in this case.
} 


\subsection{Practical considerations}

Suppose we have a sample of observed units. As noted, the one-sided derivatives $\bar{\varphi}_{+}^{\prime}(0)$ and $\bar{\varphi}_{-}^{\prime}(0)$ are correctly defined only for the units that are efficient in the direction of perturbation vector $\Delta^{B}$, i.e., satisfy Assumption 1 . If a unit does not satisfy this assumption, we may still be interested in evaluating these derivatives at the projection (5) of this unit on the production frontier.

Two scenarios arise. First, suppose that we are interested in the evaluation of the onesided derivatives $\bar{\varphi}_{+}^{\prime}(0)$ and $\bar{\varphi}_{-}^{\prime}(0)$ only at the units that satisfy Assumption 1 , and are not interested in these derivatives at the projected units. In this case, as highlighted in Section 3.3, we can simply solve programs (3) and (4) for each unit in the sample. Note that this does not require the testing of Assumption 1 at each unit. The interpretation of the optimal solutions of programs (3) and (4) is given according to one of the three possible cases discussed in Section 3.3. In particular, organising the processing of all units in this way, we detect which units are on the production frontier (in the sense of Assumption 1) and which are not. For the former units, we either identify the one-sided derivatives $\bar{\varphi}_{+}^{\prime}(0)$ and $\bar{\varphi}_{-}^{\prime}(0)$ or establish that these are undefined because the required marginal change in the direction of perturbation vector $\Delta^{A}$ is impossible in the given technology.

Alternatively, suppose that, for all units that are not on the production frontier (in the sense of Assumption 1), we are still interested in the evaluation of $\bar{\varphi}_{+}^{\prime}(0)$ and $\bar{\varphi}_{-}^{\prime}(0)$ at their projections (5) on the frontier. This task is accomplished in two separate steps. First, we evaluate the directional response function $\bar{\varphi}(0)$ for each unit in the sample. Obviously, $\bar{\varphi}(0)=0$ means that the unit is located on the production frontier and $\bar{\varphi}(0)>0$ means that the unit is not located on it. Second, according to Proposition 1, we solve programs (3) and (4) in which the constraint (3c) is replaced by equality (6). Note that, if $\bar{\varphi}(0)=0$, the original constraint $(3 \mathrm{c})$ is the same as $(6)$, so the change affects only the units that are not on the production frontier. By solving the resulting programs for each unit in the sample, we evaluate the one-sided derivatives $\bar{\varphi}_{+}^{\prime}(0)$ and $\bar{\varphi}_{-}^{\prime}(0)$ either at the unit itself (if it is located on the production frontier) or at its projection (if it is not located on the production frontier). As noted (see Footnote 4), these programs are always feasible. Therefore, the interpretation of their optimal solutions is given according to the first two cases considered in Section 3.3. Namely, for each unit or its projection we either identify the finite one-sided derivatives $\bar{\varphi}_{+}^{\prime}(0)$ and $\bar{\varphi}_{-}^{\prime}(0)$ or determine that the required marginal movement in the direction of vector $\Delta^{A}$ is impossible in the technology.

In Section 9, we consider an extended example in which we follow the second procedure described above.

\subsection{Extensions to other technologies}

Theorem 1 shows how the unifying linear programming approach of Podinovski et al. (2016) is restated for the technology $\mathcal{T}_{\mathrm{K}}$ of Kuosmanen (2005). A similar restatement can be obtained for any other polyhedral technology with undesirable outputs. Below we briefly comment on some of these technologies.

A common technology with undesirable outputs is the CRS technology which assumes joint weak disposability of the good and bad outputs, and the strong disposability of the inputs and good outputs (Chen \& Delmas, 2012; Lee, Oh, \& Lee, 2014). This technology, denoted below $\mathcal{T}^{*}$, is the cone extension of technology $\mathcal{T}_{\mathrm{K}}$. It is stated as the set of all nonnegative units $(X, V, W)$ for which there exists a vector $\lambda \in \mathbb{R}^{n}$ and slack vectors $S_{X} \in$ 
$\mathbb{R}^{m}$ and $S_{V} \in \mathbb{R}^{s}$ such that the following conditions are true:

$$
\begin{aligned}
& \bar{X} \lambda+S_{X}=X, \\
& \bar{V} \lambda-S_{V}=V, \\
& \bar{W} \lambda=W, \\
& \lambda, S_{X}, S_{V} \geq 0 .
\end{aligned}
$$

It is straightforward to verify that Theorem 1 remains valid for technology $\mathcal{T}^{*}$, with the following modifications: the variable $\omega$ and the inequalities (3e) are removed from programs (3) and (4).

Another common approach to modelling undesirable outputs is based on the assumption of their strong disposability, which is mathematically equivalent to the use of the standard CRS technology in which the undesirable inputs are treated as inputs (Färe, Grosskopf, Lovell, \& Pasurka, 1989; Mekaroonreung \& Johnson, 2012). This requires the same modification of programs (3) and (4) as for technology $\mathcal{T}^{*}$ (discussed above) and, additionally, declaring $z$ as a nonnegative vector: $z \geq 0$. A particular specification of these programs for the evaluation of different partial scale elasticity measures (see Section 6 below) applicable to the CRS technology, was discussed in detail in Atici and Podinovski (2012).

Finally, one can allow strong disposability of the undesirable outputs (i.e., treating them as inputs), in addition to the axioms A1-A3 of the technology of Kuosmanen (2005) as stated in Section 2. The resulting technology is stated in the form (1) in which the equality (1c) is changed to

$$
\bar{W} \lambda+S_{W}=W
$$

where $S_{W} \in \mathbb{R}_{+}^{p}$. In this case, the vector $z$ in programs (3) and (4) should be declared nonnegative. As noted by Kuosmanen and Podinovski (2009), this variant of technology $\mathcal{T}_{\mathrm{K}}$ is a special case of the hybrid returns-to-scale (HRS) technology of Podinovski (2004a).

\section{Marginal rates}

Theorem 1 is useful for the evaluation of various marginal rates on the production frontier. Below we consider several scenarios defined by different sets $A$ and $B$, each of which consists of a single element (input, good or bad output). For all these scenarios we restate the general programs (3) and (4) in a more specific and simpler form. ${ }^{5}$

The programs stated below are also suitable (after a straightforward modification discussed in Section 3.4) for the evaluation of marginal rates at the projections of the inefficient units on the production frontier in the direction $\Delta^{B}$.

\subsection{Rate of transformation between a bad and good output}

Consider the marginal rate of transformation $\partial V_{r^{\prime}} / \partial W_{l^{\prime}}$ of a good output $r^{\prime} \in\{1, \ldots, s\}$ with respect to a bad output $l^{\prime} \in\{1, \ldots, p\}$. Define the single-dimensional vectors $\left(\Delta_{W}^{A}\right)_{l^{\prime}}=$ 1 and $\left(\Delta_{V}^{B}\right)_{r^{\prime}}=1$. Although each of these vectors has a single component, we use the subscripts $l^{\prime}$ and $r^{\prime}$ to identify the outputs that change in the direction of these vectors.

\footnotetext{
${ }^{5}$ The programs stated in this section are restatements of programs (3) and (4) given by Theorem 1 which generally requires that Assumption 2 be satisfied. However, as shown in Appendix A, the specific programs presented in this section for the evaluation of marginal raters are valid at any unit $\left(X_{o}, V_{o}, W_{o}\right)$, even if Assumption 2 is not satisfied. The only exceptions are programs (14) and (15) that rely on Assumption 2, or its relaxed variant stated in Appendix A. If this assumption is not true at a particular unit $\left(X_{o}, V_{o}, W_{o}\right)$, we need to replace programs (14) and (15) by the appropriately specified extended programs (B.1) and (B.2) given in Appendix B.
} 
Note that we take $\left(\Delta_{V}^{B}\right)_{r^{\prime}}=1$ (and not $\left(\Delta_{V}^{B}\right)_{r^{\prime}}=-1$ ) because efficient production requires the maximization of the good output $r^{\prime}$. With vector $\left(\Delta_{V}^{B}\right)_{r^{\prime}}$ defined this way, $\left(V_{o}\right)_{r^{\prime}}+\bar{\varphi}(\gamma)\left(\Delta_{V}^{B}\right)_{r^{\prime}}=\left(V_{o}\right)_{r^{\prime}}+\bar{\varphi}(\gamma)$ is the maximum amount of the good output $r^{\prime}$ possible for the given $\gamma$, assuming that the other inputs and outputs are kept constant. Therefore, by (2), if the bad output changes from the value $\left(W_{o}\right)_{l^{\prime}}$ to $\left(W_{o}\right)_{l^{\prime}}+\gamma\left(\Delta_{W}^{A}\right)_{l^{\prime}}=\left(W_{o}\right)_{l^{\prime}}+\gamma$, the good output $r^{\prime}$, as observed on the production frontier, changes from $\left(V_{o}\right)_{r^{\prime}}$ to $\left(V_{o}\right)_{r^{\prime}}+\bar{\varphi}(\gamma)$.

The relationship between the marginal rate of transformation $\partial^{+} V_{r^{\prime}} / \partial W_{l^{\prime}}$ and the righthand derivative of the function $\bar{\varphi}(\gamma)$ is now established as follows:

$$
\begin{aligned}
\frac{\partial^{+} V_{r^{\prime}}\left(X_{o}, V_{o}, W_{o}\right)}{\partial W_{l^{\prime}}} & =\lim _{\gamma \rightarrow 0+} \frac{\left[\left(V_{o}\right)_{r^{\prime}}+\bar{\varphi}(\gamma)\right]-\left(V_{o}\right)_{r^{\prime}}}{\left[\left(W_{o}\right)_{l^{\prime}}+\gamma\right]-\left(W_{o}\right)_{l^{\prime}}} \\
& =\lim _{\gamma \rightarrow 0+} \frac{\bar{\varphi}(\gamma)}{\gamma}=\bar{\varphi}_{+}^{\prime}(0) .
\end{aligned}
$$

Using program (3) and noting that $\left(\Delta_{W}^{A}\right)^{\top} z^{A}=z_{l^{\prime}}$ and $\left(\Delta_{V}^{B}\right)^{\top} u^{B}=u_{r^{\prime}}$, we have:

$$
\begin{aligned}
& \frac{\partial^{+} V_{r^{\prime}}\left(X_{o}, V_{o}, W_{o}\right)}{\partial W_{l^{\prime}}}=\min z_{l^{\prime}} \\
& \text { s.t. } \quad u_{r^{\prime}}=1 \text { and }(3 \mathrm{c})-(3 \mathrm{f}) .
\end{aligned}
$$

Similarly, we have:

$$
\begin{aligned}
& \frac{\partial^{-} V_{r^{\prime}}\left(X_{o}, V_{o}, W_{o}\right)}{\partial W_{l^{\prime}}}=\max z_{l^{\prime}} \\
& \text { s.t. } \quad u_{r^{\prime}}=1 \text { and }(3 \mathrm{c})-(3 \mathrm{f}) .
\end{aligned}
$$

We can now use Theorem 1 and the discussion in Section 3.3 to interpret optimal solutions of programs (7) and (8) and related solver diagnostics. Namely, to be specific, if program (7) has a finite optimal solution, then the unit $\left(X_{o}, V_{o}, W_{o}\right)$ is weakly efficient with respect to the good output $r^{\prime}$ (and is in this sense located on the production frontier). The right-hand marginal rate of transformation $\partial^{+} V_{r^{\prime}} / \partial W_{l^{\prime}}$ is correctly defined at this unit and is equal to the optimal value of program (7).

If program (7) is feasible but has an unbounded optimal value, the unit $\left(X_{o}, V_{o}, W_{o}\right)$ is also weakly efficient with respect to the good output $r^{\prime}$. However, any marginal increase of the bad output $l^{\prime}$ (change in the direction $\left(\Delta_{W}^{A}\right)_{l^{\prime}}$ ) leads outside technology $\mathcal{T}_{\mathrm{K}}$. For this reason, the right-hand marginal rate of transformation $\partial^{+} V_{r^{\prime}} / \partial W_{l^{\prime}}$ is undefined.

Finally, if program (7) is infeasible (in which case program (8) is also infeasible), the unit $\left(X_{o}, V_{o}, W_{o}\right)$ is not weakly efficient with respect to the good output $r^{\prime}$ and, in this respect, is not located on the production frontier. For this reason, the right-hand marginal rate of transformation $\partial^{+} V_{r^{\prime}} / \partial W_{l^{\prime}}$ is undefined. As shown in Section 3.4, in this case we may still be interested in the one-sided marginal rates of transformation at the projection of the unit $\left(X_{o}, V_{o}, W_{o}\right)$ on the frontier obtained in the direction of good output $r^{\prime}$. According to Proposition 1, such rates are evaluated by solving programs (7) and (8) in which the constraint $(3 \mathrm{c})$ is replaced by equality (6).

Example 1 considered in Section 8 and the extended computational example in Section 9 illustrate the above discussion.

\subsection{Rate of transformation between an input and a bad output}

Consider the rate of transformation $\partial^{+} W_{l^{\prime}} / \partial X_{i^{\prime}}$ of a bad output $l^{\prime} \in\{1, \ldots, p\}$ with respect to an input $i^{\prime} \in\{1, \ldots, m\}$. Define the single-dimensional vector $\left(\Delta_{X}^{A}\right)_{i^{\prime}}=1$. 
Because efficient production of the bad output requires its minimization, and in line with Assumption 1, we define $\left(\Delta_{W}^{B}\right)_{l^{\prime}}=-1$. Then, by (2), if the input changes from the value $\left(X_{o}\right)_{i^{\prime}}$ to $\left(X_{o}\right)_{i^{\prime}}+\gamma$, the bad output, as observed on the production frontier, changes from $\left(W_{o}\right)_{l^{\prime}}$ to $\left(W_{o}\right)_{l^{\prime}}+\bar{\varphi}(\gamma)\left(\Delta_{W}^{B}\right)_{l^{\prime}}=\left(W_{o}\right)_{l^{\prime}}-\bar{\varphi}(\gamma)$. Therefore,

$$
\begin{aligned}
\frac{\partial^{+} W_{l^{\prime}}\left(X_{o}, V_{o}, W_{o}\right)}{\partial X_{i^{\prime}}} & =\lim _{\gamma \rightarrow 0+} \frac{\left[\left(W_{o}\right)_{l^{\prime}}-\bar{\varphi}(\gamma)\right]-\left(W_{o}\right)_{l^{\prime}}}{\left[\left(X_{o}\right)_{i^{\prime}}+\gamma\right]-\left(X_{o}\right)_{i^{\prime}}} \\
& =\lim _{\gamma \rightarrow 0+} \frac{-\bar{\varphi}(\gamma)}{\gamma}=-\bar{\varphi}_{+}^{\prime}(0) .
\end{aligned}
$$

Using program (3) and noting that $\left(\Delta_{X}^{A}\right)^{\top} v^{A}=v_{i^{\prime}}$ and $\left(\Delta_{W}^{B}\right)^{\top} z^{B}=-z_{l^{\prime}}$, we have

$$
\begin{aligned}
& \frac{\partial^{+} W_{l^{\prime}}\left(X_{o}, V_{o}, W_{o}\right)}{\partial X_{i^{\prime}}}=-\min v_{i^{\prime}} \\
& \text { s.t. } \quad z_{l^{\prime}}=1 \text { and }(3 \mathrm{c})-(3 \mathrm{f}) .
\end{aligned}
$$

Similarly,

$$
\begin{aligned}
& \frac{\partial^{-} W_{l^{\prime}}\left(X_{o}, V_{o}, W_{o}\right)}{\partial X_{i^{\prime}}}=-\max v_{i^{\prime}} \\
& \text { s.t. } \quad z_{l^{\prime}}=1 \text { and }(3 \mathrm{c})-(3 \mathrm{f}) .
\end{aligned}
$$

Example 2 in Section 8 illustrates the use of programs (9) and (10).

\subsection{Marginal productivity}

Consider an input $i^{\prime} \in\{1, \ldots, m\}$ and a good output $r^{\prime} \in\{1, \ldots, s\}$. Define the singledimensional vectors $\left(\Delta_{X}^{A}\right)_{i^{\prime}}=1$ and $\left(\Delta_{V}^{B}\right)_{r^{\prime}}=1$. Then

$$
\begin{aligned}
\frac{\partial^{+} V_{r^{\prime}}\left(X_{o}, V_{o}, W_{o}\right)}{\partial X_{i^{\prime}}} & =\lim _{\gamma \rightarrow 0+} \frac{\left[\left(V_{o}\right)_{r^{\prime}}+\bar{\varphi}(\gamma)\right]-\left(V_{o}\right)_{r^{\prime}}}{\left[\left(X_{o}\right)_{i^{\prime}}+\gamma\right]-\left(X_{o}\right)_{i^{\prime}}} \\
& =\lim _{\gamma \rightarrow 0+} \frac{\bar{\varphi}(\gamma)}{\gamma}=\bar{\varphi}_{+}^{\prime}(0) .
\end{aligned}
$$

Note that $\left(\Delta_{X}^{A}\right)^{\top} v^{A}=v_{i^{\prime}}$ and $\left(\Delta_{V}^{B}\right)^{\top} u^{B}=u_{r^{\prime}}$. Restating program (3), we obtain

$$
\begin{aligned}
& \frac{\partial^{+} V_{r^{\prime}}\left(X_{o}, V_{o}, W_{o}\right)}{\partial X_{i^{\prime}}}=\min v_{i^{\prime}} \\
& \text { s.t. } \quad u_{r^{\prime}}=1 \text { and }(3 \mathrm{c})-(3 \mathrm{f}) .
\end{aligned}
$$

In order to evaluate the left-hand marginal productivity $\partial^{-} V_{r^{\prime}} / \partial X_{i^{\prime}}$ at the unit $\left(X_{o}, V_{o}, W_{o}\right)$, we solve program (11) in which the minimization of $v_{i^{\prime}}$ is replaced by its maximization.

\subsection{Rate of transformation between two bad outputs}

Consider two bad outputs $l^{\prime}, l^{*} \in\{1, \ldots, p\}$. Define the single-dimensional vectors $\left(\Delta_{W}^{A}\right)_{l^{\prime}}=1$ and $\left(\Delta_{W}^{B}\right)_{l^{*}}=-1$. As above, note that the choice of vector $\left(\Delta_{W}^{B}\right)_{l^{*}}$ represents the correct direction for the bad output, as it needs to be reduced in order to project the unit on the production frontier. We then have

$$
\begin{aligned}
\frac{\partial^{+} W_{l^{*}}\left(X_{o}, V_{o}, W_{o}\right)}{\partial W_{l^{\prime}}} & =\lim _{\gamma \rightarrow 0+} \frac{\left[\left(W_{o}\right)_{l^{*}}-\bar{\varphi}(\gamma)\right]-\left(W_{o}\right)_{l^{*}}}{\left[\left(W_{o}\right)_{l^{\prime}}+\gamma\right]-\left(W_{o}\right)_{l^{\prime}}} \\
& =\lim _{\gamma \rightarrow 0+} \frac{-\bar{\varphi}(\gamma)}{\gamma}=-\bar{\varphi}_{+}^{\prime}(0) .
\end{aligned}
$$


Noting that $\left(\Delta_{W}^{A}\right)^{\top} z^{A}=z_{l^{\prime}}$ and $\left(\Delta_{W}^{B}\right)^{\top} z^{B}=-z_{l^{*}}$, we restate program (3) as follows:

$$
\begin{aligned}
& \frac{\partial^{+} W_{l^{*}}\left(X_{o}, V_{o}, W_{o}\right)}{\partial W_{l^{\prime}}}=-\min z_{l^{\prime}} \\
& \text { s.t. } \quad z_{l^{*}}=1 \text { and }(3 \mathrm{c})-(3 \mathrm{f}) .
\end{aligned}
$$

Similarly, the left-hand marginal rate of transformation $\partial^{-} W_{l^{*}} / \partial W_{l^{\prime}}$ at the unit $\left(X_{o}, V_{o}, W_{o}\right)$ is evaluated by solving program (12) whose objective $-\min z_{l^{\prime}}$ is replaced by $-\max z_{l^{\prime}}$.

\subsection{Rate of substitution between two inputs}

Consider two inputs $i^{\prime}, i^{*} \in\{1, \ldots, m\}$. Define the single-dimensional vectors $\left(\Delta_{X}^{A}\right)_{i^{\prime}}=1$ and $\left(\Delta_{X}^{B}\right)_{i^{*}}=-1$. Note that the definition of $\Delta_{X}^{B}$ is consistent with the minimization of the inputs on the production frontier. We have

$$
\begin{aligned}
\frac{\partial^{+} X_{i^{*}}\left(X_{o}, V_{o}, W_{o}\right)}{\partial X_{i^{\prime}}} & =\lim _{\gamma \rightarrow 0+} \frac{\left[\left(X_{o}\right)_{i^{*}}-\bar{\varphi}(\gamma)\right]-\left(X_{o}\right)_{i^{*}}}{\left[\left(X_{o}\right)_{i^{\prime}}+\gamma\right]-\left(X_{o}\right)_{i^{\prime}}} \\
& =\lim _{\gamma \rightarrow 0+} \frac{-\bar{\varphi}(\gamma)}{\gamma}=-\bar{\varphi}_{+}^{\prime}(0) .
\end{aligned}
$$

Restating program (3) and noting that $\left(\Delta_{X}^{A}\right)^{\top} v^{A}=v_{i^{\prime}}$ and $\left(\Delta_{X}^{B}\right)^{\top} v^{B}=-v_{i^{*}}$, we have

$$
\begin{aligned}
& \frac{\partial^{+} X_{i^{*}}\left(X_{o}, V_{o}, W_{o}\right)}{\partial X_{i^{\prime}}}=-\min v_{i^{\prime}} \\
& \text { s.t. } \quad v_{i^{*}}=1 \text { and }(3 \mathrm{c})-(3 \mathrm{f}) .
\end{aligned}
$$

Similarly, the left-hand marginal rate of substitution $\partial^{-} X_{i^{*}} / \partial X_{i^{\prime}}$ at the unit $\left(X_{o}, V_{o}, W_{o}\right)$ is evaluated by solving program $(13)$ whose objective $-\min v_{i^{\prime}}$ is replaced by $-\max v_{i^{\prime}}$.

\subsection{Rate of transformation between two good outputs}

To evaluate the marginal rate of transformation between two good outputs $r^{\prime}, r^{*} \in$ $\{1, \ldots, s\}$, define the single-dimensional vectors $\left(\Delta_{V}^{A}\right)_{r^{\prime}}=1$ and $\left(\Delta_{V}^{B}\right)_{r^{*}}=1$. We then have

$$
\begin{aligned}
\frac{\partial^{+} V_{r^{*}}\left(X_{o}, V_{o}, W_{o}\right)}{\partial V_{r^{\prime}}} & =\lim _{\gamma \rightarrow 0+} \frac{\left[\left(V_{o}\right)_{r^{*}}+\bar{\varphi}(\gamma)\right]-\left(V_{o}\right)_{r^{*}}}{\left[\left(V_{o}\right)_{r^{\prime}}+\gamma\right]-\left(V_{o}\right)_{r^{\prime}}} \\
& =\lim _{\gamma \rightarrow 0+} \frac{\bar{\varphi}(\gamma)}{\gamma}=\bar{\varphi}_{+}^{\prime}(0) .
\end{aligned}
$$

Taking into account that $\left(\Delta_{V}^{A}\right)^{\top} u^{A}=u_{r^{\prime}}$ and $\left(\Delta_{V}^{B}\right)^{\top} u^{B}=u_{r^{*}}$, we restate (3) as

$$
\begin{aligned}
& \frac{\partial^{+} V_{r^{*}}\left(X_{o}, V_{o}, W_{o}\right)}{\partial V_{r^{\prime}}}=\min \left(-u_{r^{\prime}}\right)=-\max u_{r^{\prime}} \\
& \text { s.t. } \quad u_{r^{*}}=1 \text { and }(3 \mathrm{c})-(3 \mathrm{f}) .
\end{aligned}
$$

Similarly, to estimate $\partial^{-} V_{r *} / \partial V_{r^{\prime}}$ at the $\operatorname{unit}\left(X_{o}, V_{o}, W_{o}\right)$, we solve

$$
\begin{aligned}
& \frac{\partial^{+} V_{r^{*}}\left(X_{o}, V_{o}, W_{o}\right)}{\partial V_{r^{\prime}}}=\max \left(-u_{r^{\prime}}\right)=-\min u_{r^{\prime}} \\
& \text { s.t. } \quad u_{r^{*}}=1 \text { and }(3 \mathrm{c})-(3 \mathrm{f}) .
\end{aligned}
$$




\section{Pricing undesirable outputs}

In technologies with undesirable outputs, the marginal rate of transformation $\partial V_{r^{\prime}} / \partial W_{l^{\prime}}$ is often used for the evaluation of the unobserved price $q_{l^{\prime}}$ of a bad output $l^{\prime}$ by relating the latter to the known price $p_{r^{\prime}}$ of a good output $r^{\prime}$ (Färe et al., 2005; Zhou et al., 2014). An established approach to this uses an appropriately specified directional distance function $\vec{D}(X, V, W ; g)$. In particular, it is common to use the directional vector $g=\left(g_{X}, g_{V}, g_{W}\right)$ defined as follows:

$$
\begin{array}{r}
g_{X_{i}}=0, i=1, \ldots, m, \\
g_{V_{r}}=1, r=1, \ldots, s, \\
g_{W_{l}}=-1, l=1, \ldots, p .
\end{array}
$$

It is worth noting that, unless all good and bad outputs are strongly disposable (which is not assumed in technology $\left.\mathcal{T}_{\mathrm{K}}\right)$, the function $\vec{D}(X, V, W ; g)$ with the vector $g$ defined as in (16) does not provide a complete functional representation of the technology in the sense of Chambers et al. $(1996,1998) .{ }^{6}$ This does not, however, cause problems: the representation property is not required for the correct evaluation of marginal characteristics on the production frontier and is not stated as a condition of Theorem 1.

The function $\vec{D}(X, V, W ; g)$ can be estimated by both parametric and nonparametric approaches. In the parametric approach, it is estimated in a particular class of differentiable (e.g., quadratic) functions. As shown by Färe, Grosskopf, Lovell, and Yaisawarng (1993) and Färe et al. (2005), for the unit $\left(X_{o}, V_{o}, W_{o}\right)$ we have

$$
\frac{q_{l^{\prime}}}{p_{r^{\prime}}}=-\frac{\partial \vec{D}\left(X_{o}, V_{o}, W_{o} ; g\right) / \partial W_{l^{\prime}}}{\partial \vec{D}\left(X_{o}, V_{o}, W_{o} ; g\right) / \partial V_{r^{\prime}}}=\frac{\partial V_{r^{\prime}}\left(X_{o}, V_{o}, W_{o} ; g\right)}{\partial W_{l^{\prime}}}
$$

where the second equality follows from the implicit function theorem. If the price of the good output $p_{r^{\prime}}$ is known, the unknown price of the bad output $q_{l^{\prime}}$ is found from (17).

As noted by Färe et al. (2005), if the unit $\left(X_{o}, V_{o}, W_{o}\right)$ is efficient in direction $g$, i.e., $\vec{D}\left(X_{o}, V_{o}, W_{o} ; g\right)=0$, the exact specification of the vector $g$ is unimportant. For an inefficient unit, formula (17) defines the marginal rate of transformation $\partial V_{r^{\prime}} / \partial W_{l^{\prime}}$ at its projection on the production frontier, and both depend on the chosen direction $g{ }^{7}$

The nonparametric estimation is also common in the literature - see, e.g., Chambers, Serra, and Oude Lansink (2014), Lee et al. (2014), Lee, Park, and Kim (2002), and Lee and Zhou (2015). In this approach, the function $\vec{D}(X, V, W ; g)$ is represented by the optimal value of a linear program. If the shadow prices of the constraints corresponding to the bad output $l^{\prime}$ and good output $r^{\prime}$ in this program are unique, they are equal to the partial derivatives $\partial \vec{D}(\cdot) / \partial W_{l^{\prime}}$ and $\partial \vec{D}(\cdot) / \partial V_{r^{\prime}}$ and can be used to obtain the price $q_{l^{\prime}}$ from formula (17).

\footnotetext{
${ }^{6}$ This is because the inequality $\vec{D}(X, V, W ; g) \geq 0$ does not imply that $(X, V, W) \in \mathcal{T}_{\mathrm{K}}$.

${ }^{7}$ The translation property of the function $\vec{D}(\cdot)$ (Chambers et al., 1998) implies that both partial derivatives $\partial \vec{D}(\cdot) / \partial W_{l^{\prime}}$ and $\partial \vec{D}(\cdot) / \partial V_{r^{\prime}}$ are the same if evaluated at the unit $\left(X_{o}, V_{o}, W_{o}\right)$ and at its projection. It is also important to note that, if the denominator $\partial \vec{D}(\cdot) / \partial V_{r^{\prime}} \neq 0$, the projection of the unit $\left(X_{o}, V_{o}, W_{o}\right)$ satisfies Assumption 1 by maximizing the production of output $r^{\prime}$ (which is different from maximizing the production of the vector $g$ defined by (16)), as required for the meaningful definition of the marginal rate $\partial V_{r^{\prime}}(\cdot) / \partial W_{l^{\prime}}$. Indeed, because $\vec{D}(\cdot)$ is nonincreasing in good outputs and $\partial \vec{D}(\cdot) / \partial V_{r^{\prime}} \neq 0$, we necessarily have $\partial \vec{D}(\cdot) / \partial V_{r^{\prime}}<0$. Therefore, any increase of the output $r^{\prime}$ would make the function $\vec{D}(\cdot)$ at the resulting unit strictly negative, which proves that the maximum of output $r^{\prime}$ has been achieved.
} 
A known problem with this approach is that the shadow prices of the good and bad outputs, evaluated at strongly efficient observed units, and therefore their ratio in (17), are usually not unique (see, e.g., Førsund, 2018). This raises the question of identifying the minimal and maximal values of this ratio that would translate into the lower and upper bounds $q_{l^{\prime}}^{\min }$ and $q_{l^{\prime}}^{\max }$ on the price of the bad output. Note that computing the maximal and minimal values of $\partial \vec{D}(\cdot) / \partial W_{l^{\prime}}$ and $\partial \vec{D}(\cdot) / \partial V_{r^{\prime}}$, which can be done by solving two additional linear programs for each of the two partial derivatives, is insufficient for this purpose.

In our paper, using technology $\mathcal{T}_{\mathrm{K}}$ as an example, we show that the above problem is easily resolved by evaluating the one-sided partial derivatives $\partial^{+} V_{r^{\prime}} / \partial W_{l^{\prime}}$ and $\partial^{-} V_{r^{\prime}} / \partial W_{l^{\prime}}$ directly, by solving linear programs (7) and (8). Let the unit $\left(X_{o}, V_{o}, W_{o}\right)$ be efficient in the production of the good output $r^{\prime}$, i.e., satisfy Assumption 1. Taking into account that program (7) minimizes the objective function $z_{l^{\prime}}$ and (8) maximizes it, we always have

$$
\frac{\partial^{+} V_{r^{\prime}}\left(X_{o}, V_{o}, W_{o}\right)}{\partial W_{l^{\prime}}} \leq \frac{\partial^{-} V_{r^{\prime}}\left(X_{o}, V_{o}, W_{o}\right)}{\partial W_{l^{\prime}}}
$$

provided both partial derivatives exist. Theorem 1 and the discussion in Sections 3.3 and 4.1 provide a complete interpretation of the optimal solutions and related solver diagnostics that can theoretically arise in the evaluation of these derivatives.

Using (17) and suppressing the now redundant notation for vector $g$, we have:

$$
q_{l^{\prime}}^{\min }=\frac{\partial V_{r^{\prime}}^{+}\left(X_{o}, V_{o}, W_{o}\right)}{\partial W_{l^{\prime}}} p_{r^{\prime}}, \quad q_{l^{\prime}}^{\max }=\frac{\partial V_{r^{\prime}}^{-}\left(X_{o}, V_{o}, W_{o}\right)}{\partial W_{l^{\prime}}} p_{r^{\prime}}
$$

The suggested approach based on solving programs (7) and (8) is a variant of the approach expressed by formula (17). Indeed, these programs effectively employ the directional distance function $\vec{D}(X, V, W ; g)$, where $g=e_{r^{\prime}}$ is the orthonormal vector corresponding to dimension $r^{\prime}$. With this specification, the shadow price $\partial \vec{D}(\cdot) / \partial V_{r^{\prime}}$ in formula (17) is unique and is equal to 1 (as explicitly required by the first constraint $u_{r^{\prime}}=1$ of programs $(7)$ and (8)), and the numerator $\partial \vec{D}(\cdot) / \partial W_{l^{\prime}}$ is equal to $\partial V_{r^{\prime}}(\cdot) / \partial W_{l^{\prime}}$. As a side note unimportant for the evaluation of marginal rates, let us mention that, because the good output $r^{\prime}$ is strongly disposable, the directional distance function with $g=e_{r^{\prime}}$ completely characterizes technology $\mathcal{T}_{\mathrm{K}}$ in the sense of Chambers et al. $(1996,1998)$.

If the unit $\left(X_{o}, V_{o}, W_{o}\right)$ is inefficient in the production of the good output $r^{\prime}$, i.e., if it does not satisfy Assumption 1, both programs (7) and (8) need a straightforward modification, as discussed in Section 3.4. As follows from Proposition 1, in this case, the one-sided partial derivatives $\partial^{+} V_{r^{\prime}} / \partial W_{l^{\prime}}$ and $\partial^{-} V_{r^{\prime}} / \partial W_{l^{\prime}}$ are evaluated at the projection of the unit $\left(X_{o}, V_{o}, W_{o}\right)$ on the production frontier in the direction of maximization of the good output $r^{\prime}$. Note that this projection is generally different from the projection obtained in the direction of the vector $g$ defined by (16). Therefore, for an inefficient unit, the one-sided derivatives evaluated at its projection will generally be different depending on the direction $g$ used in the definition of the directional distance function.

As discussed in Section 3.6, programs similar to (7) and (8) can be stated for any other polyhedral technology with bad outputs. It might be worth emphasising again that this is equivalent to the use of the directional distance function defined by the vector $g$, with the single positive component $g_{V_{r^{\prime}}}=1$ corresponding to the good output $r^{\prime}$ which is used for the pricing of the bad output.

The computational example considered in Section 9 provides a further illustration to the above discussion. 


\section{Elasticity measures}

In this section we show how the main Theorem 1 can be restated for the evaluation of the elasticity of proportional response of the measures in the set $B$ with respect to marginal proportional changes of the measures in the set $A$, while keeping the measures in the set $C$ constant. The full and partial scale elasticities are examples of such elasticity measures.

\subsection{Definitions and main results}

We start with the following definition, which is a multiplicative analogue of Definition 2.

Definition 3. For the unit $\left(X_{o}, V_{o}, W_{o}\right) \in \mathcal{T}_{\mathrm{K}}$ and the sets $A, B$ and $C$, the proportional response function $\bar{\beta}(\alpha)$ is the optimal value of the following linear program in which $\alpha$ is a parameter:

$$
\begin{array}{r}
\bar{\beta}(\alpha)=\max \left\{\beta \mid\left(\alpha X_{o}^{A}, \beta X_{o}^{B}, X_{o}^{C}, \alpha V_{o}^{A}, \beta V_{o}^{B}, V_{o}^{C},\right.\right. \\
\left.\left.\alpha W_{o}^{A}, \beta W_{o}^{B}, W_{o}^{C}\right) \in \mathcal{T}_{\mathrm{K}}, \beta \in \mathbb{R}\right\} .
\end{array}
$$

Program (18) has a clear meaning. For example, if $B$ is the set of all good output, $A$ is the set of all inputs and bad outputs, and the set $C$ is empty, program (18) evaluates the maximum $\bar{\beta}(\alpha)$ of the expansion factor $\beta$ for the good outputs that keeps the resulting unit in technology $\mathcal{T}_{\mathrm{K}}$, provided all inputs and bad outputs are proportionally changed by $\alpha$.

Similar to the previous development of marginal rates, the notion of elasticity measures (including the scale elasticity) is well-defined only for the units on the production frontier. The following assumption is a direct analogue of Assumption 1.

Assumption 3. The unit $\left(X_{o}, V_{o}, W_{o}\right)$ is weakly efficient with respect to the proportional increase of the measures in the set $B$, i.e., $\bar{\beta}(1)=1$.

Assumption 3 requires that the unit $\left(X_{o}, V_{o}, W_{o}\right)$ be on the production frontier in the sense that no proportional increase (with $\beta>1$ ) of the measures in the set $B$ is possible. For example, if the set $B$ is the set of all good outputs, then the unit $\left(X_{o}, V_{o}, W_{o}\right)$ is output radial efficient with respect to the good outputs.

Assuming that the required one-sided derivatives exist (see Theorem 2 below), define the one-sided elasticities of response of the measures in the set $B$ with respect to marginal proportional changes of the measures in the set $A$, evaluated at the unit $\left(X_{o}, V_{o}, W_{o}\right)$, as follows:

$$
\varepsilon_{A, B}^{+}\left(X_{o}, V_{o}, W_{o}\right)=\bar{\beta}_{+}^{\prime}(1), \quad \varepsilon_{A, B}^{-}\left(X_{o}, V_{o}, W_{o}\right)=\bar{\beta}_{-}^{\prime}(1) .
$$

In the conventional convex technology without undesirable outputs, and with $A$ and $B$ being the sets of all inputs and outputs, respectively, the above approach results in the definition of the one-sided estimates $\varepsilon_{A, B}^{+}\left(X_{o}, V_{o}, W_{o}\right)$ and $\varepsilon_{A, B}^{-}\left(X_{o}, V_{o}, W_{o}\right)$ of the conventional scale elasticity. This approach to the definition of elasticity measures as the derivatives of the proportional response function $\bar{\beta}(\alpha)$ is consistent with the definitions of Frisch (1965), Starrett (1977), Førsund (1996) and Førsund and Hjalmarsson (2004). Definition 3 of the function $\bar{\beta}(\alpha)$ in the setting described by the three subsets $A, B$ and $C$ was first suggested by Podinovski and Førsund (2010) and further explored by Podinovski et al. (2016) and Podinovski (2017).

Let us show that the one-sided elasticity measures (19) can be calculated using Theorem 1. Indeed, the proportional response function $\bar{\beta}(\alpha)$ can be redefined as a special case of the directional response function $\bar{\varphi}(\gamma)$ defined by $(2)$. Let

$$
\begin{aligned}
& \left(\Delta_{X}^{A}, \Delta_{V}^{A}, \Delta_{W}^{A}\right)=\left(X_{o}^{A}, V_{o}^{A}, W_{o}^{A}\right), \\
& \left(\Delta_{X}^{B}, \Delta_{V}^{B}, \Delta_{W}^{B}\right)=\left(X_{o}^{B}, V_{o}^{B}, W_{o}^{B}\right) .
\end{aligned}
$$


Then, according to (2),

$$
\begin{aligned}
\bar{\varphi}(\gamma)=\max \{\varphi \mid & \left((1+\gamma) X_{o}^{A},(1+\varphi) X_{o}^{B}, X_{o}^{C},\right. \\
& (1+\gamma) V_{o}^{A},(1+\varphi) V_{o}^{B}, V_{o}^{C}, \\
& \left.\left.(1+\gamma) W_{o}^{A},(1+\varphi) W_{o}^{B}, W_{o}^{C}\right) \in \mathcal{T}_{\mathrm{K}}, \varphi \in \mathbb{R}\right\}
\end{aligned}
$$

Denote $\alpha=1+\gamma$ and $\beta=1+\varphi$. Comparing (2) with (20), we have

$$
\bar{\beta}(\alpha)=1+\bar{\varphi}(\alpha-1) .
$$

Differentiating by $\alpha$ and taking into account (19), we have

$$
\begin{aligned}
& \varepsilon_{A, B}^{+}\left(X_{o}, V_{o}, W_{o}\right)=\bar{\beta}_{+}^{\prime}(1)=\bar{\varphi}_{+}^{\prime}(0), \\
& \varepsilon_{A, B}^{-}\left(X_{o}, V_{o}, W_{o}\right)=\bar{\beta}_{-}^{\prime}(1)=\bar{\varphi}_{-}^{\prime}(0) .
\end{aligned}
$$

This simple relationship allows us to restate Theorem 1 for the case of one-sided elasticity measures (19). We first define $\tilde{\Gamma}$ as the domain of the function $\bar{\beta}(\alpha){ }^{8}$ We also note that Assumption 2 is no longer required, i.e., the next result applies to all units in technology $\mathcal{T}_{\mathrm{K}}$.

Theorem 2. Let $\left(X_{o}, V_{o}, W_{o}\right)$ be any unit in technology $\mathcal{T}_{\mathrm{K}}$.

1. If Assumption 3 is satisfied then the following four statements are true:

(i) If $\alpha=1$ is not the right extreme point of domain $\tilde{\Gamma}$, then the right-hand elasticity of response $\varepsilon_{A, B}^{+}(\cdot)=\varepsilon_{A, B}^{+}\left(X_{o}, V_{o}, W_{o}\right)$ exists, is finite and is equal to the optimal value of the linear program

$$
\begin{aligned}
& \varepsilon_{A, B}^{+}(\cdot)=\min \left(X_{o}^{A}\right)^{\top} v^{A}-\left(V_{o}^{A}\right)^{\top} u^{A}+\left(W_{o}^{A}\right)^{\top} z^{A} \\
& \text { s.t. }-\left(X_{o}^{B}\right)^{\top} v^{B}+\left(V_{o}^{B}\right)^{\top} u^{B}-\left(W_{o}^{B}\right)^{\top} z^{B}=1, \\
& X_{o}^{\top} v-V_{o}^{\top} u+W_{o}^{\top} z+\omega=0, \\
& \bar{X}^{\top} v-\bar{V}^{\top} u+\bar{W} z+1 \omega \geq 0, \\
& \bar{X}^{\top} v+1 \omega \geq 0, \\
& u, v \geq 0, z, \omega \operatorname{sign} \text { free. }
\end{aligned}
$$

(ii) If $\alpha=1$ is the right extreme point of $\tilde{\Gamma}$, then the optimal value of program (22) is unbounded.

(iii) If $\alpha=1$ is not the left extreme point of $\tilde{\Gamma}$, then the left-hand elasticity of response $\varepsilon_{A, B}^{-}(\cdot)=\varepsilon_{A, B}^{-}\left(X_{o}, V_{o}, W_{o}\right)$ exists, is finite and is equal to the optimal value of the linear program

$$
\begin{aligned}
& \varepsilon_{A, B}^{-}(\cdot)=\max \left(X_{o}^{A}\right)^{\top} v^{A}-\left(V_{o}^{A}\right)^{\top} u^{A}+\left(W_{o}^{A}\right)^{\top} z^{A} \\
& \text { s.t. }(22 \mathrm{~b})-(22 \mathrm{f}) .
\end{aligned}
$$

(iv) If $\alpha=1$ is the left extreme point of $\tilde{\Gamma}$, then the optimal value of program (23) is unbounded.

2. If Assumption 3 is not satisfied, the programs (22) and (23) are infeasible.

\footnotetext{
${ }^{8}$ Taking into account $(21), \bar{\beta}(\alpha)$ is defined for all $\alpha$ such that $\alpha-1$ is in the domain $\Gamma$ of the function $\bar{\varphi}(\alpha)$. Therefore, $\tilde{\Gamma}=\{\alpha \mid \alpha-1 \in \Gamma\}$.
} 
In practical applications, programs (22) and (23) may have finite or unbounded optimal values, or be infeasible. As discussed in Section 3.3, if, for example, program (22) has a finite optimal solution, then the unit $\left(X_{o}, V_{o}, W_{o}\right)$ satisfies Assumption 3, the right-hand elasticity of response $\varepsilon_{A, B}^{+}\left(X_{o}, V_{o}, W_{o}\right)$ is correctly defined and is equal to the optimal value of (22). If the optimal value of program (22) is unbounded, then the unit $\left(X_{o}, V_{o}, W_{o}\right)$ satisfies Assumption 3 but any marginal proportional increase of the input and output measures in the set $A$ leads outside technology $\mathcal{T}_{\mathrm{K}}$. For this reason, the right-hand elasticity measure $\varepsilon_{A, B}^{+}\left(X_{o}, V_{o}, W_{o}\right)$ is undefined. Finally, if program (22) is infeasible, then the unit $\left(X_{o}, V_{o}, W_{o}\right)$ does not satisfy Assumption 3 and the elasticity measure $\varepsilon_{A, B}^{+}\left(X_{o}, V_{o}, W_{o}\right)$ is undefined. Similar analysis applies to program (23).

\subsection{Elasticity measures for projections of inefficient units}

Suppose that the unit $\left(X_{o}, V_{o}, W_{o}\right)$ does not satisfy Assumption 3, i.e., we have $\bar{\beta}(1)>1$, where $\bar{\beta}(1)$ is evaluated by solving linear program (18) with $\alpha=1$. In this case, the one-sided elasticity measures $\varepsilon_{A, B}^{+}(\cdot)$ and $\varepsilon_{A, B}^{-}(\cdot)$ are undefined. However, we may still be interested in evaluating such elasticity measures at the projection

$$
\begin{aligned}
\left(X^{*}, V^{*}, W^{*}\right)= & \left(X_{o}^{A}, \bar{\beta}(1) X_{o}^{B}, X_{o}^{C},\right. \\
& V_{o}^{A}, \bar{\beta}(1) V_{o}^{B}, V_{o}^{C}, \\
& \left.W_{o}^{A}, \bar{\beta}(1) W_{o}^{B}, W_{o}^{C}\right) .
\end{aligned}
$$

of the unit $\left(X_{o}, V_{o}, W_{o}\right)$ on the production frontier obtained by the proportional increase of the measures in the set $B$ by the factor $\bar{\beta}(1)>1$. Such projection satisfies Assumption 3 and we can use Theorem 2 for the calculation of the one-sided elasticity measures $\varepsilon_{A, B}^{+}\left(X^{*}, V^{*}, W^{*}\right)$ and $\varepsilon_{A, B}^{-}\left(X^{*}, V^{*}, W^{*}\right)$.

More specifically, let $\bar{\beta}^{*}(\alpha)$ be the proportional response function (18) defined at the projection $\left(X^{*}, V^{*}, W^{*}\right)$, and let $\tilde{\Gamma}^{*}$ be its domain. The required one-sided elasticities $\varepsilon_{A, B}^{+}\left(X^{*}, V^{*}, W^{*}\right)$ and $\varepsilon_{A, B}^{-}\left(X^{*}, V^{*}, W^{*}\right)$ are now obtained by solving programs (22) and (23) in which the unit $\left(X_{o}, V_{o}, W_{o}\right)$ is replaced by its projection $\left(X^{*}, V^{*}, W^{*}\right)$.

An alternative and equivalent way of evaluating the one-sided elasticity measures at the projection $\left(X^{*}, V^{*}, W^{*}\right)$ is given by the following result. Its advantage is that it equally applies to all units, regardless of whether they satisfy Assumption 3. If the unit $\left(X_{o}, V_{o}, W_{o}\right)$ satisfies Assumption 3, then $\bar{\beta}(1)=1$ and this result is just a restatement of Theorem 2 .

Proposition 2. The domains $\tilde{\Gamma}^{*}$ and $\tilde{\Gamma}$ of the functions $\bar{\beta}^{*}(\alpha)$ and $\bar{\beta}(\alpha)$ coincide. The statements 1(i)-1(iv) of Theorem 2 remain valid for the projected unit $\left(X^{*}, V^{*}, W^{*}\right)$ with the following two modifications to programs (22) and (23). ${ }^{9}$ First, the constraint (22c) is replaced by the equality

$$
X_{o}^{\top} v-V_{o}^{\top} u+W_{o}^{\top} z+\omega=\bar{\beta}(1)-1
$$

Second, the elasticity measures $\varepsilon_{A, B}^{+}\left(X^{*}, V^{*}, W^{*}\right)$ and $\varepsilon_{A, B}^{-}\left(X^{*}, V^{*}, W^{*}\right)$ are obtained by dividing the optimal values of the objective functions in the resulting modified programs (22) and (23) by $\bar{\beta}(1)$, respectively.

We illustrate the use of Proposition 2 in Example 3 considered in Section 8.

\footnotetext{
${ }^{9}$ Because the projected unit satisfies Assumption 3, the statement 2 of Theorem 2 does not apply.
} 


\section{Special cases of elasticity measures}

The general methodology for the calculation of one-sided elasticities of response in technology $\mathcal{T}_{\mathrm{K}}$ is based on programs (22) and (23) and their interpretation by Theorem 2. Different scenarios that might be of interest in practical applications correspond to different definitions of the sets $A, B$ and $C$. Below we consider two such scenarios.

\subsection{Elasticity of response of good outputs to changes of all inputs and bad outputs}

Let the set $A$ include all inputs and all bad outputs, and let the set $B$ include all good outputs. In this case, the set $C$ is empty and program (22) is restated as follows:

$$
\begin{aligned}
\varepsilon_{A, B}^{+}(\cdot)=\min & X_{o}^{\top} v+W_{o}^{\top} z \\
\text { s.t. } & V_{o}^{\top} u=1, \\
& X_{o}^{\top} v-V_{o}^{\top} u+W_{o}^{\top} z+\omega=0, \\
& \bar{X}^{\top} v-\bar{V}^{\top} u+\bar{W} z+1 \omega \geq 0, \\
& \bar{X}^{\top} v+1 \omega \geq 0, \\
& u, v \geq 0, z, \omega \text { sign free. }
\end{aligned}
$$

Similarly, we have

$$
\begin{aligned}
\varepsilon_{A, B}^{-}(\cdot)=\max & X_{o}^{\top} v+W_{o}^{\top} z \\
\text { s.t. } & (26 \mathrm{~b})-(26 \mathrm{f}) .
\end{aligned}
$$

Taking into account equalities (26b) and (26c), restate the objective function of programs $(26)$ and $(27)$ as $1-\omega$. Define $\omega^{\max }=\max \{\omega \mid(26 \mathrm{~b})-(26 \mathrm{f})\}$ and $\omega^{\min }=\min \{\omega \mid$ $(26 \mathrm{~b})-(26 \mathrm{f})\}$. Restating programs (26) and (27) in terms of $\omega^{\max }$ and $\omega^{\min }$, we have

$$
\varepsilon_{A, B}^{+}(\cdot)=1-\omega^{\max }, \quad \varepsilon_{A, B}^{-}(\cdot)=1-\omega^{\min } .
$$

\subsection{Elasticity of response of good outputs to changes of all inputs}

In this scenario we evaluate the elasticity of proportional response of the full vector of good outputs with respect to marginal proportional changes of the full vector of inputs, while keeping the vector of bad outputs constant. Let the set $A$ include all inputs, the set $B$ include all good outputs and the set $C$ include all bad outputs. Then programs (22) and (23) are restated as follows:

$$
\begin{aligned}
\varepsilon_{A, B}^{+}(\cdot)=\min & X_{o}^{\top} v \\
\text { s.t. } & (26 \mathrm{~b})-(26 \mathrm{f}),
\end{aligned}
$$

and

$$
\begin{aligned}
\varepsilon_{A, B}^{-}(\cdot)=\max & X_{o}^{\top} v \\
\text { s.t. } & (26 \mathrm{~b})-(26 \mathrm{f}) .
\end{aligned}
$$


Table 1: The data set.

\begin{tabular}{cccc}
\hline Unit & Input $X$ & Good output $V$ & Bad output $W$ \\
\hline$A$ & 1 & 1 & 1 \\
$B$ & 2 & 1.5 & 2 \\
$C$ & 5 & 1 & 0.25 \\
$D$ & 4 & 1 & 0.4375 \\
\hline
\end{tabular}

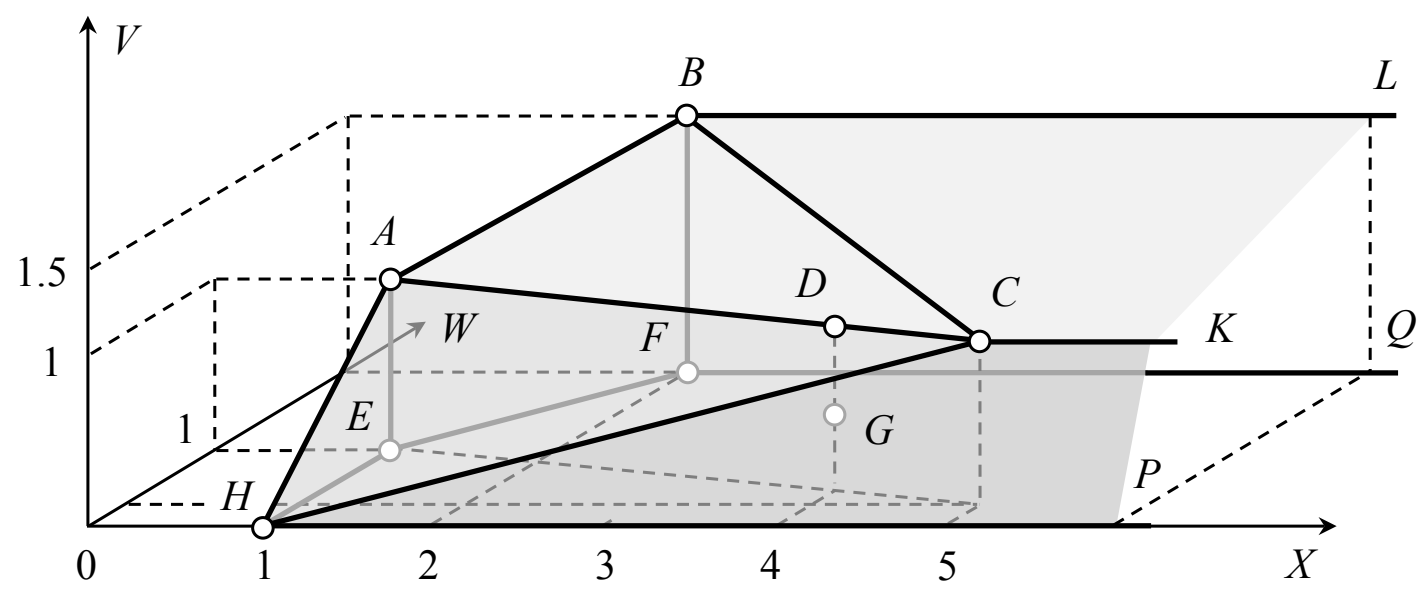

Figure 2: Technology $\mathcal{T}_{\mathrm{K}}$ generated by units $A, B$ and $C$.

\section{Examples}

Below we consider three examples illustrating the usefulness of the theoretical approach developed in this paper. In all of these examples, we use the same technology $\mathcal{T}_{\mathrm{K}}$ generated by the three observed units $A, B$ and $C$ shown in Table 1 . (The unit $D$ is not observed but is used in the discussion below.) This technology is shown in Fig. 2.

Note that the point $H$ is obtained by the joint weak disposal of the good and bad outputs of the unit $A$. The facets $A B C$ and $A C H$ are included in the technology by the assumption of convexity. The unbounded facets $L B C K$ and $K C H P$ represent strong disposability of the input. The facets $A E H, A B F E$ and $L B F Q$ are included by the assumption of strong disposability of the good output.

Example 1. Consider the evaluation of the marginal rate of transformation between the bad and good outputs $W$ and $V$ at the units $A, B, C$ and $D$. Note that all these units satisfy Assumption 2. Therefore, we can formally use Theorem 1 in which programs (3) and (4) are replaced by programs (7) and (8), respectively. ${ }^{10}$

\footnotetext{
${ }^{10}$ As shown in Footnote 5, for the marginal rate of transformation between the bad and good output, Theorem 1 remains true even if Assumption 2 is not satisfied.
} 
Table 2: One-sided marginal rates $\partial^{-} W / \partial V$ and $\partial^{+} W / \partial V$ in Example 1.

\begin{tabular}{ccc}
\hline Unit & $\partial^{-} V / \partial W$ & $\partial^{+} V / \partial W$ \\
\hline$A$ & 1 & $-\infty$ \\
$B$ & 0.4211 & $-\infty$ \\
$C$ & 4 & 0.2857 \\
$D$ & 1 & 0.4211 \\
\hline
\end{tabular}

For example, for the unit $D$, program (7) is stated as follows:

$$
\begin{aligned}
\frac{\partial^{+} V}{\partial W}(D)= & \min \quad z \\
\text { s.t. } \quad & u=1 \\
& 4 v-u+0.4375 z+\omega=0, \\
& v-u+z+\omega \geq 0, \\
& 2 v-1.5 u+2 z+\omega \geq 0, \\
& 5 v-u+0.25 z+\omega \geq 0, \\
& v+\omega \geq 0 \\
& 2 v+\omega \geq 0 \\
& 5 v+\omega \geq 0 \\
& u, v \geq 0, \quad z, \omega \text { sign free. }
\end{aligned}
$$

Similarly, for the evaluation of the left-hand marginal rate $\partial^{-} V / \partial W(D)$, we use program (8). This requires changing the minimization of $z$ in program (28) to its maximization. ${ }^{11}$

For the evaluation of the right-hand marginal rate of transformation at the other units, we need to replace only the equality (28c). For example, for unit $B$, this constraint takes on the form:

$$
2 v-1.5 u+2 z+\omega=0 .
$$

Table 2 shows the results of computations. The interpretation of these results for different units is similar, and we comment only on the results for the unit $A$.

The left-hand rate of transformation $\partial^{-} V / \partial W(A)$ is equal to 1 and represents the slope of the line $A H$ (along which the input $X$ is kept constant). Solving program (7) for the right-hand rate of transformation $\partial^{+} V / \partial W(A)$ results in the unbounded optimal value of this program, reported as $-\infty$. As follows from Theorem 1 and as discussed in Sections 3.3 and 4.1, this means that any marginal increase of the bad output $W$ of the unit $A$, while keeping the input $X$ fixed, leads outside the technology, and for this reason $\partial^{+} V / \partial W(A)$ is undefined. This result is also clear from Fig. 2.

Example 2. Consider the evaluation of the marginal rate of transformation between the input $X$ and the bad output $W$ using programs (9) and (10). For example, for the unit $D$,

\footnotetext{
${ }^{11}$ In a similar example considered by Podinovski et al. (2016), the values of the bad output enter the linear programs with the negative sign. Because $z$ is a sign-free variable, the two approaches are equivalent.
} 
Table 3: One-sided marginal rates $\partial^{-} W / \partial X$ and $\partial^{+} W / \partial X$ in Example 2.

\begin{tabular}{ccc}
\hline Unit & $\partial^{-} W / \partial X$ & $\partial^{+} W / \partial X$ \\
\hline$A$ & $-\infty$ & -0.1875 \\
$B$ & $-\infty$ & 0 \\
$C$ & -0.1875 & 0 \\
$D$ & -0.1875 & -0.1875 \\
\hline
\end{tabular}

the former program takes on the form:

$$
\begin{aligned}
& \frac{\partial^{+} W}{\partial X}(D)=-\min \quad v \\
& \text { s.t. } \quad z=1 \text { and }(28 \mathrm{c})-(28 \mathrm{j}) .
\end{aligned}
$$

For the left-hand marginal rate $\partial^{-} W / \partial X(D)$, we solve program (10) which requires changing the minimization of $v$ in program (29) to its maximization. The evaluation for the other units is based on similar programs that require changing only the constraint (28c).

The results of computations in this scenario are shown in Table 3. Note that the lefthand marginal rates $\partial^{-} W / \partial X$ at the units $A$ and $B$ are undefined because any decrease of the input $X$ at these units (while keeping the good output $V$ fixed) leads outside the technology. This results in an unbounded value of program (10) stated for these two units.

In all the other cases, the one-sided rates of transformation $\partial^{-} W / \partial X$ and $\partial^{+} W / \partial X$ are either negative or equal to zero. Because these rates are evaluated by keeping the good output $V$ fixed, this means that an increase of the input in technology $\mathcal{T}_{\mathrm{K}}$ can not result in an increase of the bad output. For example, consider unit $D$. Changing its input $X$ and keeping the good output $V$ constant and equal to 1 makes the bad output $W$ change so that the resulting unit is located on the edge $A C$. According to Table 3 , an increase or reduction of the input by a small value $\delta$ leads to the reduction or, respectively, increase of the bad output by $0.1875 \delta$, so that there is a negative relationship between the two measures.

It is also interesting to note that the right-hand rates of transformation $\partial^{+} W / \partial X$ evaluated at the units $B$ and $C$ are equal to zero. For example, increasing the input $X$ of the unit $C$ while keeping the good output $V$ fixed, forces the bad output $W$ to remain unchanged (as this is required to maintain efficiency), so that the resulting unit moves along the ray $C K$ away from $C$.

Example 3. Consider assessing the elasticity of response of the good output with respect to simultaneous proportional changes of the input and the bad output. Define $A=\{X, W\}$ and $B=\{V\}$. In this case we use Theorem 2 in which, according to the described scenario, we replace its generic programs (22) and (23) by programs (26) and (27), respectively.

For example, for the unit $D$, program (26) takes on the form:

$$
\begin{aligned}
\varepsilon_{A, B}^{+}(D)= & \min \quad 4 v+0.4375 z \\
\text { s.t. } & u=1 \text { and }(28 \mathrm{c})-(28 \mathrm{j}) .
\end{aligned}
$$

Similarly, to assess $\varepsilon_{A, B}^{-}(D)$, we change the minimization of the objective function in (30) by its maximization.

Table 4 shows the results of computations in this case. Note that the two cases of unbounded solutions correctly identify the situations in which the corresponding one-sided 
Table 4: One-sided elasticities of response $\varepsilon_{A, B}^{-}$and $\varepsilon_{A, B}^{+}$in Example 3.

\begin{tabular}{ccc}
\hline Unit & $\varepsilon_{A, B}^{-}$ & $\varepsilon_{A, B}^{+}$ \\
\hline$A$ & $+\infty$ & 0.5 \\
$B$ & 0.6667 & $-\infty$ \\
$C$ & 1.1875 & 0.0714 \\
$D$ & 1.1875 & 0.5 \\
\hline
\end{tabular}

elasticity of response is undefined. For example, the left-hand elasticity of response $\varepsilon_{A, B}^{-}$ at the unit $A$ is undefined because any marginal proportional change of the input and bad output leads outside the technology. Similarly, the right-hand measure $\varepsilon_{A, B}^{+}$at the unit $B$ is undefined because any marginal proportional increase of the input and bad output results in an infeasible combination.

Finally, let us illustrate Proposition 2. Consider unit $G$ obtained from unit $D$ by reducing the level of its good output from 1 to 0.5. Unit $G$ does not satisfy Assumption 3. The good output of this unit can be increased by the factor 2 , i.e., we have $\bar{\beta}(1)=2$. The projection of the unit $G$ in this case is unit $D$. To evaluate the elasticity measures at the projection using Proposition 2, we first solve the minimization program (30) and its maximization analogue in which the zero on the right-hand side of constraint (28c) is replaced by $\bar{\beta}(1)-1=2-1=1$, i.e., by replacing (28c) by the equality

$$
4 v-u+0.4375 z+\omega=1 .
$$

Calculations show that the optimal values of these programs are equal to 1 and 2.375, respectively. Next, according to Proposition 2, we divide these optimal values by $\bar{\beta}(1)=2$. This produces the one-sided elasticities $\varepsilon_{A, B}^{+}=0.5$ and $\varepsilon_{A, B}^{-}=1.1875$ evaluated at the projected unit $D$. Note that these values coincide with the one-sided elasticity measures evaluated directly at unit $D$, by solving program (30) and its maximization analogues, as shown in Table 4.

\section{Computational example}

Below we consider an example of a polluting technology with two inputs, one good and two bad outputs, based on simulated data. This example illustrates the use of the direct approach to the estimation of one-sided derivatives of a good output with respect to a bad output which, as discussed in Section 5, is often used for the estimation of the unobserved price of the bad output. This example also highlights the known (Førsund, 2018) limitations of the traditional approach based on the ratio (17) of shadow prices of the bad and good outputs obtained in the evaluation of the conventionally specified directional distance function.

We consider the following simple data generating process (DGP). Input $X_{1}$ is randomly generated from the uniform distribution $U[10,50]$. Input $X_{2}$ is considered to be a polluting input (e.g., $X_{2}$ may represent fossil fuel used in electricity production). It is obtained by the formula $X_{2}=\xi X_{1}$, where $\xi_{1}$ is generated from $U[0.1,1.1]$. We assume that the maximum (efficient) production $\bar{V}_{1}$ of the good output is given by the formula $\bar{V}_{1}=X_{1}^{0.5} X_{2}^{0.4}$. To introduce inefficiency, we randomly generate the output efficiency $\xi_{2}$ from $U[0.7,1]$ and define the actual production of the good output as $V_{1}=\xi_{2} \bar{V}_{1}$. We assume that the two bad outputs $W_{1}$ and $W_{2}$ depend on the input $X_{2}$ as follows: $W_{1}=\xi_{3} X_{2}$ and $W_{2}=\xi_{4} X_{2}$, 
where $\xi_{3} \sim U[0.5,0.6]$ and $\xi_{4} \sim U[0.1,0.3]$. We finally round the data to two decimal points. Table 5 shows a sample of 45 units generated by the described DGP.

Consider estimating the marginal rate of transformation $\partial V_{1} / \partial W_{1}$ between the bad output $W_{1}$ and the good output $V_{1}$ in technology $\mathcal{T}_{\mathrm{K}}$ generated by the above sample of units. As shown in Section 4.1, this requires the evaluation of the one-sided derivatives $\bar{\varphi}_{+}^{\prime}(0)$ and $\bar{\varphi}_{-}^{\prime}(0)$ of the function $\bar{\varphi}(\gamma)$ defined by the single-dimensional vectors of perturbation with components $\left(\Delta_{W}^{A}\right)_{1}=1$ and $\left(\Delta_{V}^{B}\right)_{1}=1$.

Following the second procedure outlined in Section 3.5, we first evaluate the optimal value $\bar{\varphi}(0)$ at each unit in our sample, by solving linear program (2) in which $\gamma=0$. The results are shown in the second column of Table 6. (All results in Table 6 are rounded to four decimal places.) Note that $\bar{\varphi}(0)=0$ if and only if the unit under the consideration is efficient in the direction of vector $\Delta^{B}=\Delta_{V}^{B}$. Because we have only one good output, this assumption is equivalent to the condition that the unit is output radial efficient. ${ }^{12}$

The one-sided marginal rates $\partial^{+} V_{1} / \partial W_{1}$ and $\partial^{-} V_{1} / \partial W_{1}$ are correctly defined only at the units that satisfy Assumption 1, i.e., at the units for which $\bar{\varphi}(0)=0$. In this case they are evaluated by Theorem 1 or, more specifically, by solving programs (7) and (8), as illustrated in Example 1. If a unit is inefficient in the direction of the good output $V_{1}$, i.e., if $\bar{\varphi}(0)>0$, we use Proposition 1 in order to evaluate the one-sided rates of transformation at the output projection of the inefficient unit on the boundary of the technology.

More specifically, we solve programs (7) and (8) for each of the 45 units in the sample, in which, by Proposition 1, we replace the zero on the right-hand side of equality $(3 \mathrm{c})$ by $\bar{\varphi}(0)$. (This means that we actually solve unmodified programs (7) and (8) for all units that satisfy Assumption 1.) For example, for Unit 2, we have $\bar{\varphi}(0)=1.7063$. Therefore, we evaluate the right-hand marginal rate $\partial^{+} V_{1} / \partial W_{1}$ at the output projection of this unit by solving the following linear program: ${ }^{13}$

$$
\begin{aligned}
& \frac{\partial^{+} V_{1}}{\partial W_{1}}(\text { Unit } 2)=\min z_{1} \\
& \text { s.t. } \quad u_{1}=1 \\
& 23.61 v_{1}+13.35 v_{2}-11.03 u_{1}+7.79 z_{1}+3.27 z_{2}+\omega=1.7063 \\
& 27.88 v_{1}+4.24 v_{2}-8.38 u_{1}+2.5 z_{1}+0.82 z_{2}+\omega \geq 0 \\
& 23.61 v_{1}+13.35 v_{2}-11.03 u_{1}+7.79 z_{1}+3.27 z_{2}+\omega \geq 0 \\
& \ldots \\
& 33.86 v_{1}+37.1 v_{2}-19.89 u_{1}+20.61 z_{1}+7.56 z_{2}+\omega \geq 0 \\
& 27.88 v_{1}+4.24 v_{2}+\omega \geq 0 \\
& 23.61 v_{1}+13.35 v_{2}+\omega \geq 0 \\
& \ldots \\
& 33.86 v_{1}+37.1 v_{2}+\omega \geq 0 \\
& u_{1}, v_{1}, v_{2} \geq 0, z_{1}, z_{2}, \omega \text { sign free. }
\end{aligned}
$$

Similarly, the left-hand marginal rate of transformation $\partial^{-} V_{1} / \partial W_{1}$ is assessed by changing the minimization of $z_{1}$ in the above program to its maximization.

\footnotetext{
${ }^{12}$ In the case of several good outputs, the output radial efficiency should be assessed only with respect to the selected good output, by keeping the other good outputs unchanged.

${ }^{13}$ For the actual computations, instead of the rounded value $\bar{\varphi}(0)=1.7063$ shown in the second constraint, we use the original (not rounded) value of $\bar{\varphi}(0)$ as found by the solver.
} 
The third and fourth columns of Table 6 show the results of computations of the one-sided marginal rates of transformation. For any unit that satisfies Assumption 1, i.e., such that the corresponding $\bar{\varphi}(0)=0$, these marginal rates are evaluated at the unit itself. However, if $\bar{\varphi}(0)>0$, then, by Proposition 1, the two marginal rates are evaluated at the output projection of the unit on the boundary of the technology.

In Table 6 , we use the symbols $-\infty$ and $+\infty$ to identify the cases in which the optimal values of the minimization and maximization programs (7) and (8) are unbounded. By Theorem 1 (see also Section 3.3) the case of $-\infty$ means that the bad output $W_{1}$ of a particular unit cannot be increased in technology $\mathcal{T}_{\mathrm{K}}$, while keeping the remaining inputs and outputs constant. For this reason, the right-hand marginal rate $\partial^{+} V_{1} / \partial W_{1}$ is undefined. Similarly, in the case of $+\infty$, the bad output of the unit cannot be reduced and the left-hand marginal rate $\partial^{-} V_{1} / \partial W_{1}$ is undefined. Note that, for several units (e.g., for Units 4 and 11), we simultaneously have $\partial^{+} V_{1} / \partial W_{1}=-\infty$ and $\partial^{-} V_{1} / \partial W_{1}=+\infty$. This means that we can neither increase nor reduce the bad output $W_{1}$ of such units while keeping the other inputs and outputs fixed, and therefore neither one-sided marginal rate is defined.

For comparison, we also implement the conventional approach based on the directional distance function $\vec{D}(\cdot)=\vec{D}(X, V, W ; g)$ defined by the directional vector $g=\left(g_{X}, g_{V}, g_{W}\right)$ as in (16). The last four columns of Table 6 show the value of $\vec{D}(\cdot)$, the shadow prices $\partial \vec{D}(\cdot) / \partial V_{1}$ and $\partial \vec{D}(\cdot) / \partial W_{1}$ of the good and bad outputs (as reported by the solver) and the marginal rate of transformation calculated by formula (17). As noted in Section 5, if $\vec{D}(\cdot)>0$, the marginal rate of transformation is evaluated at the projection of the given unit on the boundary of technology obtained in the direction of vector $g=\left(g_{X}, g_{V}, g_{W}\right)$. Also note that, for the Units 10, 27 and 33, the required ratio is undefined because its denominator is equal to zero.

Let us first consider the results obtained for the units for which both $\bar{\varphi}(0)=0$ and $\vec{D}(\cdot)=0$. In this case, both approaches evaluate the marginal rate of transformation between the good and bad outputs at the same observed unit, and not at its projections (which are generally different for inefficient units). Overall, 23 units satisfy this condition.

It is straightforward to verify that, for all of such units, the marginal rate $\partial V_{1} / \partial W_{1}$ estimated using the shadow prices of the directional distance function is always within the range between the one-sided partial derivatives $\partial^{+} V_{1} / \partial W_{1}$ and $\partial^{-} V_{1} / \partial W_{1}$ obtained by the direct approach based on Theorem 1 . Because the shadow prices of the bad and good outputs are generally not unique, the marginal rate of transformation calculated by formula (17) is not uniquely defined. Our computations demonstrate that such rate (shown in the last column of Table 6) is just one arbitrary representative of an often very wide range between the minimal and maximal possible rates $\partial^{+} V_{1} / \partial W_{1}$ and $\partial^{-} V_{1} / \partial W_{1}$. For example, for Unit 3 , the marginal rate estimated by the ratio of shadow prices is 0.4114 , which coincides with $\partial^{-} V_{1} / \partial W_{1}$. However, for Unit 44, the marginal rate -0.9849 coincides with $\partial^{+} V_{1} / \partial W_{1}$.

For the remaining 22 units we have either $\bar{\varphi}(0)>0$ or $\vec{D}(\cdot)>0$, or both. ${ }^{14}$ For these units, the direct method and the method based on the use of shadow prices estimate the marginal rate of transformation at the projected units. Because the projections for the two methods are generally different, the corresponded estimates of the marginal rates are unrelated and should not be compared to each other.

\footnotetext{
${ }^{14}$ For technology $\mathcal{T}_{\mathrm{K}}$, the equality $\vec{D}(\cdot)=0$ implies $\bar{\varphi}(0)=0$, but the opposite is not true. This fact can also be observed from Table 6 . To prove this, suppose that, for some unit $\left(X_{o}, V_{o}, W_{o}\right)$, we have $\bar{\varphi}(0)>0$. Then $\left(X_{o},(1+\bar{\varphi}(0)) V_{o}, W_{o}\right) \in \mathcal{T}_{\mathrm{K}}$. Using weak output disposability, reduce all good and bad outputs by some proportion $\psi<1$ such that $\psi(1+\bar{\varphi}(0))>1$. Then $\left(X_{o}, \psi(1+\bar{\varphi}(0)) V_{o}, \psi W_{o}\right) \in \mathcal{T}_{\mathrm{K}}$. Taking into account (16), we have $\vec{D}(\cdot)>0$.
} 
It is also interesting to note that, for all units for which $\bar{\varphi}(0)>0$, the one-sided marginal rates $\partial^{+} V_{1} / \partial W_{1}$ and $\partial^{-} V_{1} / \partial W_{1}$ in Table 6 are equal. (For example, for Unit 29, both are equal to 0.6843.) This is not surprising because the projections of observed units typically occur at the relative interiors of some facets of the production frontier where the function $\bar{\varphi}(\gamma)$ is differentiable and, therefore, its one-sided derivatives are equal to each other.

\section{Conclusion}

A number of different models of production technologies incorporating undesirable outputs have been suggested in the literature. A conventional approach to the evaluation of marginal characteristics of the production frontiers of such technologies is based on the use of shadow prices of the inputs and outputs for an appropriately specified directional distance function of Chambers et al. (1998). It is common to define this function by the directional vector that seeks a simultaneous increase of all good outputs and reduction of all bad outputs. Then, for example, the partial derivative of a good output with respect to a bad output (useful for the estimation of the unobserved price of the bad output) is obtained as the negated ratio of the shadow prices of the two outputs.

In the nonparametric framework, the directional distance function is estimated as the optimal value of a linear program. In this setting, estimating marginal characteristics based on the shadow prices has a well-known drawback (Førsund, 2018). Namely, the shadow prices of the inputs and outputs are typically not unique. While it is relatively easy to evaluate their minimal and maximal values (by solving additional linear programs), the evaluation of the minimal and maximal ratio of such shadow prices (actually needed for the evaluation of the marginal characteristic of interest) leads to a much less attractive nonlinear program.

In this paper, we show that the above drawback can be overcome by selecting the directional distance function in a particular way that depends on the marginal characteristic of interest. For example, for the evaluation of the marginal rate of transformation of a good output with respect to a bad output, we define this function by the directional vector with the single positive component equal to 1 , which corresponds to the good output in question.

The main theoretical result of this paper, from which different marginal characteristics are derived as special cases, is stated as Theorem 1. It is an adaptation of the unifying linear programming approach to the evaluation of marginal characteristics in arbitrary polyhedral technologies developed by Podinovski et al. (2016). The latter approach was developed in the framework of sensitivity analysis in linear programming and followed the earlier approaches of Podinovski et al. (2009) and Podinovski and Førsund (2010). This approach can also be seen as a specific operational implementation of the known results of nondifferentiable optimization whose application in DEA was pioneered by Chambers and Färe (2008).

Our paper contributes to the literature in a number of ways. Most importantly, we show that various marginal characteristics that have traditionally been evaluated as the ratio of shadow prices for the conventionally specified directional distance function can be evaluated directly. In this approach, the one-sided marginal characteristic of interest at the given frontier unit is obtained as the optimal value of a single linear program.

The suggested direct approach produces the exact minimal and maximal (or, depending on the specification, right-hand and left-hand) values of the marginal characteristic of interest, in a computationally efficient way that requires solving a single linear program for each value. This method also allows a rigorous identification and interpretation of a common situation in which the marginal perturbation embedded in the notion of marginal rate is infeasible, i.e., leads outside the technology. In such cases, the marginal rate is undefined. 
By Theorem 1, this situation is diagnosed by the unbounded solution to the corresponding linear program.

In this paper we also clarify a theoretical issue underpinning the definition and evaluation of different marginal rates. It is often assumed that, for the marginal rate to be correctly defined, the unit under the consideration should be strongly efficient and that the directional distance function used for the evaluation should be a complete functional representation of the technology in the sense of Chambers et al. (1998). We show that neither is required. In particular, we point out that the conventional directional distance function based on the simultaneous increase of the good outputs and reduction of bad outputs, does not completely characterize a weakly disposable technology. This is, however, unimportant. The only requirement for the correct definition of a marginal characteristic and its evaluation is stated as Assumption 1. This requires that the unit under the evaluation be efficient in the direction of the vector that defines the directional distance function.

In order to be specific, in this paper we state our main results for the technology of Kuosmanen (2005). The choice of this technology is not essential. As demonstrated, all presented results are easily adapted for any other polyhedral technology incorporating undesirable outputs. 


\section{Appendix A. Variants of Assumption 2}

Below we obtain a relaxed variant of Assumption 2 which is still sufficient for Theorem 1 to remain valid. We also consider this assumption for the special case arising in the evaluation of marginal rates discussed in Section 4.

Assumption 4. All good outputs of the unit $\left(X_{o}, V_{o}, W_{o}\right)$ included in the set $A$ are strictly positive, i.e., $V_{o r}>0$, for all $r \in A$. If the set $B$ includes two or more elements, then all good outputs of the unit $\left(X_{o}, V_{o}, W_{o}\right)$ included in the set $B$ are strictly positive, i.e., if $|B| \geq 2$ then $V_{\text {or }}>0$, for all $r \in B$.

Note that, if the set $B$ consists of a single element and this element is a good output, Assumption 4 does not require that this output be positive at the unit $\left(X_{o}, V_{o}, W_{o}\right)$.

Proposition 3. Theorem 1 remains valid under the weaker Assumption 4 used instead of Assumption 2.

Now consider a special case (arising in the evaluation of marginal rates in Section 4) in which each set $A$ and $B$ contains a single element (input, good or bad output). Then the requirement of Assumption 4 regarding the set $B$ is redundant and is replaced by the simpler requirement as follows.

Assumption 5. Let each set $A$ and $B$ consist of a single element. If the single element of $A$ is a good output $r$, then this output of the unit $\left(X_{o}, V_{o}, W_{o}\right)$ is strictly positive, i.e., $V_{\text {or }}>0$.

In the considered scenario involving single-element sets $A$ and $B$, Proposition 3 is restated as follows.

Proposition 4. If the sets $A$ and $B$ consist of single elements, Theorem 1 remains valid under Assumption 5 used instead of Assumption 2.

Note that, for all marginal rates discussed in Section 4, except for the marginal rate of transformation between two good outputs, the set $A$ does not contain a good output and, therefore, Assumption 5 is trivially satisfied at any unit $\left(X_{o}, V_{o}, W_{o}\right) \in \mathcal{T}_{\mathrm{K}}$, with any nonnegative (including zero) components. By Proposition 4 , the programs given in Section 4 for the evaluation of these marginal rates are valid at all units in the technology.

The only exception is the evaluation of the marginal rate of transformation between two good outputs by programs (14) and (15). If the good output $r^{\prime} \in A$ of the unit $\left(X_{o}, V_{o}, W_{o}\right)$ is equal to zero, then this unit does not satisfy Assumption 5. The required one-sided marginal rates at this unit are correctly evaluated by using programs (B.1) and (B.2) given in the statement of the general Theorem 3 in Appendix B. We illustrate the use of these two programs in Examples 4 and 5 in Appendix B. 


\section{Appendix B. Theorem 1 in the general case}

Below we extend Theorem 1 and Proposition 1 to the general case that does not involve the simplifying Assumption 2. We also present two examples that illustrate the importance of Assumption 2 (and the equivalent relaxed Assumption 4) for the validity of Theorem 1.

In the linear programs (B.1) and (B.2) stated below, the variable vectors $\sigma$ and $\zeta$ are of the same dimensions as the corresponding vectors $V_{o}^{A}$ (and $\Delta_{V}^{A}$ ) and $V_{o}^{B}$ (and $\Delta_{V}^{B}$ ).

Theorem 3. Let $\left(X_{o}, V_{o}, W_{o}\right)$ be any unit in technology $\mathcal{T}_{\mathrm{K}}$.

1. If Assumption 1 is satisfied then the following four statements are true:

(i) If $\gamma=0$ is not the right extreme point of domain $\Gamma$, then the right-hand derivative $\bar{\varphi}_{+}^{\prime}(0)$ exists, is finite and is equal to the optimal value of the following program:

$$
\begin{aligned}
& \bar{\varphi}_{+}^{\prime}(0)=\min \left(\Delta_{X}^{A}\right)^{\top} v^{A}-\left(\Delta_{V}^{A}\right)^{\top} u^{A}+\left(\Delta_{W}^{A}\right)^{\top} z^{A}+\left(\Delta_{V}^{A}\right)^{\top} \sigma \\
& \text { s.t. }-\left(\Delta_{X}^{B}\right)^{\top} v^{B}+\left(\Delta_{V}^{B}\right)^{\top} u^{B}-\left(\Delta_{W}^{B}\right)^{\top} z^{B}-\left(\Delta_{V}^{B}\right)^{\top} \zeta=1, \\
& X_{o}^{\top} v-V_{o}^{\top} u+W_{o}^{\top} z+\omega+\left(V_{o}^{A}\right)^{\top} \sigma+\left(V_{o}^{B}\right)^{\top} \zeta=0, \\
& \bar{X}^{\top} v-\bar{V}^{\top} u+\bar{W} z+1 \omega \geq 0, \\
& \bar{X}^{\top} v+1 \omega \geq 0, \\
& u, v, \sigma, \zeta \geq 0, z, \omega \operatorname{sign} \text { free. }
\end{aligned}
$$

(ii) If $\gamma=0$ is the right extreme point of $\Gamma$, then the optimal value of program (B.1) is unbounded.

(iii) If $\gamma=0$ is not the left extreme point of $\Gamma$, then the left-hand derivative $\bar{\varphi}_{-}^{\prime}(0)$ exists, is finite and is equal to the optimal value of the following program:

$$
\begin{aligned}
& \bar{\varphi}_{-}^{\prime}(0)=\max \left(\Delta_{X}^{A}\right)^{\top} v^{A}-\left(\Delta_{V}^{A}\right)^{\top} u^{A}+\left(\Delta_{W}^{A}\right)^{\top} z^{A}+\left(\Delta_{V}^{A}\right)^{\top} \sigma \\
& \text { s.t. }(\text { B.1b })-(\text { B.1f }) .
\end{aligned}
$$

(iv) If $\gamma=0$ is the left extreme point of $\Gamma$, then the optimal value of program (B.2) is unbounded.

2. If Assumption 1 is not satisfied, the programs (B.1) and (B.2) are infeasible.

If the unit $\left(X_{o}, V_{o}, W_{o}\right)$ does not satisfy Assumption 1 then, similar to the case considered in Section 3, we can still evaluate the marginal frontier characteristics at its projection $\left(X^{*}, V^{*}, W^{*}\right)$ defined by (5). The evaluation of the one-sided derivatives $\bar{\varphi}^{*^{\prime}}(0)$ and $\bar{\varphi}^{*^{\prime}}(0)$ at the projected unit is based on the following result, analogous to Proposition 1.

Proposition 5. The domains $\Gamma^{*}$ and $\Gamma$ of the functions $\bar{\varphi}^{*}(\gamma)$ and $\bar{\varphi}(\gamma)$ coincide. The statements 1(i)-1(iv) of Theorem 3 remain valid for the projected unit $\left(X^{*}, V^{*}, W^{*}\right)$ with the following modification. The one-sided derivatives $\bar{\varphi}_{*_{+}^{\prime}}(0)$ and $\bar{\varphi}^{*^{\prime}}{ }_{-}(0)$ evaluated at the projected unit $\left(X^{*}, V^{*}, W^{*}\right)$ are obtained by solving programs (B.1) and (B.2) in which the value 0 on the right-hand side of constraint (B.1c) is replaced by $\bar{\varphi}(0)$.

In the following two examples, Assumption 2 and the weaker Assumptions 4 and 5 are not satisfied. As a result, Theorem 1 is not valid, and we need to employ Theorem 3. 
Example 4. Let technology $\mathcal{T}_{\mathrm{K}}$ be generated by the observed units $A$ and $B$ shown in Table B.7. Consider the evaluation of the one-sided marginal rates of transformation $\partial^{+} W / \partial V_{2}$ and $\partial^{-} W / \partial V_{2}$ between the good and bad outputs $V_{2}$ and $W$.

Define the single-dimensional perturbation vectors with components $\left(\Delta_{V}^{A}\right)_{2}=1$ and $\left(\Delta_{W}^{B}\right)_{1}=-1$. Note that $\Delta_{W}^{B}$ identifies the direction in which the bad output needs to change in order to project the unit on the production frontier. For the chosen direction $\Delta^{B}=\Delta_{W}^{B}$, both units $A$ and $B$ satisfy Assumption 1 .

Similar to the proofs provided in Section 4, it is straightforward to show that

$$
\frac{\partial^{+} W}{\partial V_{2}}\left(X_{o}, V_{o}, W_{o}\right)=-\bar{\varphi}_{+}^{\prime}(0), \quad \frac{\partial^{-} W}{\partial V_{2}}\left(X_{o}, V_{o}, W_{o}\right)=-\bar{\varphi}_{-}^{\prime}(0) .
$$

Note that unit $A$ satisfies Assumption 5 but unit $B$ does not satisfy any of the Assumptions 2, 4 or 5. Therefore, programs (3) and (4) of Theorem 1 can be used only for unit $A$ but not for $B$. In the latter case we need to use the more general programs (B.1) and (B.2) of Theorem 3. For example, using program (B.2) and noting (B.3), we have:

$$
\begin{aligned}
\frac{\partial^{-} W}{\partial V_{2}}(B)= & -\max -u_{2}+\sigma_{2} \\
\text { s.t. } \quad & z=1, \\
& v-u_{1}+z+\omega+\sigma_{2}=0, \\
& v-u_{1}+z+\omega \geq 0, \\
& 0.5 v-1.5 u_{1}-u_{2}+2 z+\omega \geq 0, \\
& v+\omega \geq 0, \\
& 0.5 v+\omega \geq 0, \\
& v, u_{1}, u_{2}, \sigma_{2} \geq 0, z, \omega \text { sign free. }
\end{aligned}
$$

The above program has an unbounded solution. By Theorem 3, and in line with the discussion in Section 3.3, this implies that the left-hand partial derivative $\partial^{-} W / \partial V_{2}(B)$ is undefined. This is because any reduction of the good output $V_{2}$ of unit $B$, while keeping its input $X$ and good output $V_{1}$ fixed, leads outside the technology $\mathcal{T}_{\mathrm{K}}$.

Table B.8 shows both one-sided marginal rates at the units $A$ and $B$.

Suppose that we have (incorrectly) used program (4) (and negated its optimal value) instead of solving program (B.4). This is equivalent to removing the variable $\sigma_{2}$ from the latter program. The optimal value of the resulting program is equal to 0 , which is incorrect.

Example 5. Consider the same technology $\mathcal{T}_{\mathrm{K}}$ as in Example 4. Let us evaluate the onesided marginal rates of response of the vector of good outputs with respect to marginal changes of the bad output. Define the scalar $\Delta_{W}^{A}=1$ and the vector $\Delta_{V}^{B}=(1,1)$. Let $\bar{\varphi}(\gamma)$ be the directional response function defined by (2).

In this setting, unit $A$ satisfies Assumption 2 but unit $B$ does not. The latter unit also does not satisfy the relaxed Assumption 4. Therefore, we can use programs (3) and (4) of Theorem 1 only for unit $A$. For unit $B$, we should use programs (B.1) and (B.2) of Theorem 3. For example, program (B.2) for unit $B$ takes on the form:

$$
\begin{aligned}
\bar{\varphi}_{-}^{\prime}(0)= & \max z \\
\text { s.t. } & u_{1}+u_{2}-\zeta_{1}-\zeta_{2}=1, \\
& v-u_{1}+z+\omega+\zeta_{1}=0, \\
& \text { constraints }(\mathrm{B} .4 \mathrm{~d})-(\mathrm{B} .4 \mathrm{~g}), \\
& v, u_{1}, u_{2}, \zeta_{1}, \zeta_{2} \geq 0, z, \omega \text { sign free. }
\end{aligned}
$$


The above program has an unbounded value which is interpreted as discussed in Section 3.3. Namely, the left-hand marginal rate of response $\bar{\varphi}_{-}^{\prime}(0)$ is undefined because any reduction of the bad output, while keeping the input $X$ fixed, leads outside the technology. Table B.9 shows the one-sided derivatives $\bar{\varphi}_{+}^{\prime}(0)$ and $\bar{\varphi}_{-}^{\prime}(0)$ evaluated at the units $A$ and $B$ using Theorem 3.

Suppose that we have used program (4) instead of program (B.5). This is equivalent to removing the variables $\zeta_{1}$ and $\zeta_{2}$ from the latter program. Solving the resulting program, we obtain the incorrect result $\bar{\varphi}_{-}^{\prime}(0)=1$.

\section{Appendix C. Proofs}

We first prove the most general Theorem 3. The proofs of the other statements are based on this theorem and are outlined afterwards.

Proof of Theorem 3. We first note that Theorem 1 proved in Podinovski et al. (2016) is valid for any polyhedral technology, including technology $\mathcal{T}_{\mathrm{K}}$. This theorem does not depend on the classification of measures into inputs and outputs. In particular, in this theorem, we can view bad outputs as a part of the output vector $\tilde{Y}=(V, W)$ or as a part of the input vector $\tilde{X}=(X, W)$, and both approaches lead to programs (B.1) and (B.2) for the evaluation of $\bar{\varphi}_{+}^{\prime}(0)$ and $\bar{\varphi}_{-}^{\prime}(0)$. Below we only briefly outline the logic of the proof of Theorem 1 of Podinovski et al. (2016) adapted to technology $\mathcal{T}_{\mathrm{K}}$.

Let $\left(X_{o}, V_{o}, W_{o}\right)$ be any unit in technology $\mathcal{T}_{\mathrm{K}}$. (We do not require Assumption 2 or the weaker Assumption 4.) Restate (2) without the vectors $S_{X}$ and $S_{V}$ used in (1) as follows:

$$
\begin{aligned}
\bar{\varphi}(\gamma)=\max & \varphi \\
\text { s.t. } & \bar{X}^{A} \lambda+\bar{X}^{A} \mu \leq X_{o}^{A}+\gamma \Delta_{X}^{A}, \\
& \bar{X}^{B} \lambda+\bar{X}^{B} \mu-\varphi \Delta_{X}^{B} \leq X_{o}^{B}, \\
& \bar{X}^{C} \lambda+\bar{X}^{C} \mu \leq X_{o}^{C} \\
& -\bar{V}^{A} \lambda \leq-V_{o}^{A}+\gamma\left(-\Delta_{V}^{A}\right), \\
& -\bar{V}^{B} \lambda+\varphi \Delta_{V}^{B} \leq-V_{o}^{B}, \\
& -\bar{V}^{C} \lambda \leq-V_{o}^{C} \\
& \bar{W}^{A} \lambda=W_{o}^{A}+\gamma \Delta_{W}^{A} \\
& \bar{W}^{B} \lambda-\varphi \Delta_{W}^{B}=W_{o}^{B}, \\
& \bar{W}^{C} \lambda=W_{o}^{C} \\
& 1^{\top} \lambda+1^{\top} \mu=1, \\
& D=V_{o}^{A}+\gamma \Delta_{V}^{A}, \\
& -\varphi \Delta_{V}^{B} \leq V_{o}^{B} \\
& \lambda, \mu, D \geq 0, \varphi \text { sign free. }
\end{aligned}
$$

The above program is feasible if and only if the unit $\left(X_{o}, V_{o}, W_{o}\right)$ perturbed by the vectors $\gamma \Delta^{A}$ and $\varphi \Delta^{B}$ is in technology $\mathcal{T}_{\mathrm{K}}$ and, in particular, is nonnegative. The nonnegativity of the perturbed components $X_{o}^{A}+\gamma \Delta_{X}^{A}, X_{o}^{B}+\varphi \Delta_{X}^{B}, W_{o}^{A}+\gamma \Delta_{W}^{A}$ and $W_{o}^{B}+\varphi \Delta_{W}^{B}$ follows from constraints (C.1b), (C.1c), (C.1h) and (C.1i). However, the nonnegativity of the terms $V_{o}^{A}+\gamma \Delta_{V}^{A}$ and $V_{o}^{B}+\varphi \Delta_{V}^{B}$ does not follow from (C.1e) and (C.1f), and is stated explicitly by constraints (C.1l) and (C.1m), where $D$ is a nonnegative vector. 
Let $v=\left(v^{A}, v^{B}, v^{C}\right), u=\left(u^{A}, u^{B}, u^{C}\right)$ and $z=\left(z^{A}, z^{B}, z^{C}\right)$ be the dual vectors to constraints (C.1b) $-($ C.1d), (C.1e) $-($ C.1g) and (C.1h) $-($ C.1j), respectively. Let the sign-free scalar $\omega$ be dual to (C.1k). Finally, let $\sigma$ and $\zeta$ be the dual vectors to (C.1l) and (C.1m).

Consider the dual to (C.1) with $\gamma=0$. Note that the nonnegativity of vector $\sigma$ follows from the dual constraint to the primal vector $D$.

$$
\begin{aligned}
& \bar{\varphi}(0)=\min X_{o}^{\top} v-V_{o}^{\top} u+W_{o}^{\top} z+\omega+\left(V_{o}^{A}\right)^{\top} \sigma+\left(V_{o}^{B}\right)^{\top} \zeta \\
& \text { s.t. }-\left(\Delta_{X}^{B}\right)^{\top} v^{B}+\left(\Delta_{V}^{B}\right)^{\top} u^{B}-\left(\Delta_{W}^{B}\right)^{\top} z^{B}-\left(\Delta_{V}^{B}\right)^{\top} \zeta=1, \\
& \bar{X}^{\top} v-\bar{V}^{\top} u+\bar{W} z+1 \omega \geq 0, \\
& \bar{X}^{\top} v+1 \omega \geq 0, \\
& u, v, \sigma, \zeta \geq 0, z, \omega \text { sign free. }
\end{aligned}
$$

Using known results of sensitivity analysis in linear programming as in Podinovski et al. (2016), the right-hand derivative $\bar{\varphi}_{+}^{\prime}(0)$ is found as the optimal value of the following program, where the combined vector $\left(\Delta_{X}^{A},-\Delta_{V}^{A}, \Delta_{W}^{A}, \Delta_{V}^{A}\right)$ describes the vector of perturbations (used in proportion $\gamma$ ) on the right-hand side of program (C.1):

$$
\begin{gathered}
\bar{\varphi}_{+}^{\prime}(0)=\min \left\langle\left(\Delta_{X}^{A},-\Delta_{V}^{A}, \Delta_{W}^{A}, \Delta_{V}^{A}\right),\left(v^{A}, u^{A}, z^{A}, \sigma\right)\right\rangle \\
\text { s.t. }(v, u, z, \omega, \sigma, \zeta) \text { is optimal in }(\mathrm{C} .2) .
\end{gathered}
$$

By Assumption 1, $\bar{\varphi}(0)=0$. Then program (C.3) includes all constraints of program (C.2) and the additional equality requiring that the objective function of program (C.2) be equal to 0 . This produces program (B.1). Similarly, the left-hand derivative $\bar{\varphi}_{-}^{\prime}(0)$ is evaluated by changing the minimization of the objective function in (C.3) to its maximization. This leads to program (B.2).

The remaining proof of all individual statements of Theorem 3 repeats the proof of Theorem 1 in Podinovski et al. (2016), and is not given.

Proof of Theorem 1. This theorem is a special case of Theorem 3. Its proof is similar to the proof of Proposition 4 in Podinovski et al. (2016) and is only outlined. Using complementary slackness conditions, we first prove that, at any optimal solution to program (B.1) and (B.2), we have $\left(V_{o}^{A}\right)^{\top} \sigma=0$ and $\left(V_{o}^{B}\right)^{\top} \zeta=0$. By Assumption 2, we then have $\sigma=\zeta=0$, and programs (B.1) and (B.2) become programs (3) and (4), respectively.

Proof of Proposition 1. This result follows from the more general Proposition 5.

Proof of Theorem 2. The proof of this theorem is based on Theorem 3. It is similar to the proof of Proposition 5 in Podinovski et al. (2016) and is not given.

Proof of Proposition 2. A pair $\left(\alpha^{\prime}, \beta^{\prime}\right)$ is feasible in program (18) if and only if the pair $\left(\alpha^{\prime}, \beta^{\prime} / \bar{\beta}(1)\right)$ is feasible in a similar program (18) restated for the projected unit $\left(X^{*}, V^{*}, W^{*}\right)$. Therefore, $\tilde{\Gamma}^{*}=\tilde{\Gamma}$.

Consider program (22) for the evaluation of $\varepsilon_{A, B}^{+}(\cdot)=\varepsilon_{A, B}^{+}\left(X^{*}, V^{*}, W^{*}\right)$. Stating this program for the unit $\left(X^{*}, V^{*}, W^{*}\right)$ as in (24), replacing (22c) by the sum of $(22 \mathrm{~b})$ and $(22 \mathrm{c})$ 
and rearranging the terms, we have its equivalent statement

$$
\begin{aligned}
\varepsilon_{A, B}^{+}(\cdot)=\min & \left(X_{o}^{A}\right)^{\top} v^{A}-\left(V_{o}^{A}\right)^{\top} u^{A}+\left(W_{o}^{A}\right)^{\top} z^{A} \\
\text { s.t. } & \bar{\beta}(1)\left[-\left(X_{o}^{B}\right)^{\top} v^{B}+\left(V_{o}^{B}\right)^{\top} u^{B}-\left(W_{o}^{B}\right)^{\top} z^{B}\right]=1, \\
& \left(X_{o}^{A}\right)^{\top} v^{A}+\left(X_{o}^{C}\right)^{\top} v^{C}-\left(V_{o}^{A}\right)^{\top} u^{A}-\left(V_{o}^{C}\right)^{\top} u^{C} \\
& +\left(W_{o}^{A}\right)^{\top} z^{A}+\left(W_{o}^{C}\right)^{\top} z^{C}+\omega=1, \\
& \text { and }(22 \mathrm{~d})-(22 \mathrm{f}) .
\end{aligned}
$$

Also, following the statement of Proposition 2, consider program (22) in which equality (22c) is replaced by (25). Furthermore, replace (25) by its sum with (22b). Rearranging the terms and denoting the optimal value $\tilde{\varepsilon}$, we have the following equivalent statement

$$
\begin{aligned}
\tilde{\varepsilon}=\min & \left(X_{o}^{A}\right)^{\top} v^{A}-\left(V_{o}^{A}\right)^{\top} u^{A}+\left(W_{o}^{A}\right)^{\top} z^{A} \\
\text { s.t. } & -\left(X_{o}^{B}\right)^{\top} v^{B}+\left(V_{o}^{B}\right)^{\top} u^{B}-\left(W_{o}^{B}\right)^{\top} z^{B}=1, \\
& \left(X_{o}^{A}\right)^{\top} v^{A}+\left(X_{o}^{C}\right)^{\top} v^{C}-\left(V_{o}^{A}\right)^{\top} u^{A}-\left(V_{o}^{C}\right)^{\top} u^{C} \\
& +\left(W_{o}^{A}\right)^{\top} z^{A}+\left(W_{o}^{C}\right)^{\top} z^{C}+\omega=\bar{\beta}(1), \\
& \text { and }(22 \mathrm{~d})-(22 \mathrm{f}) .
\end{aligned}
$$

Note that $\left(u^{\prime}, v^{\prime}, z^{\prime}, \omega^{\prime}\right)$ is feasible in (C.4) if and only if $\left(\bar{\beta}(1) u^{\prime}, \bar{\beta}(1) v^{\prime}, \bar{\beta}(1) z^{\prime}, \bar{\beta}(1) \omega^{\prime}\right)$ is feasible in (C.5). The corresponding value of the objective function in (C.5) is equal to $\bar{\beta}(1)$ times the value of the objective function in (C.4). Therefore, for the optimal values, we have $\varepsilon_{A, B}^{+}\left(X^{*}, V^{*}, W^{*}\right)=\tilde{\varepsilon} / \bar{\beta}(1)$. The proof in the case of $\varepsilon_{A, B}^{-}\left(X^{*}, V^{*}, W^{*}\right)$ is similar and is not given.

Proof of Proposition 3. Let $|B|=1$ and let the single element of $B$ be the good output $r$. Because $\varphi$ in program (C.1) is maximized, the inequality (C.1f) implies that, at optimality, $V_{o}^{B}+\varphi \Delta_{V}^{B}=\bar{V}^{B} \lambda \geq 0$. Therefore, constraint (C.1m) is redundant and can be omitted. Then the dual variable $\zeta$ is omitted from (C.2), (B.1) and (B.2). If $|B| \geq 2$, then, by Assumption 4 , all good outputs in the set $B$ are strictly positive. The fact that the vectors $\zeta$ and $z$ can be omitted is now proved by complementary slackness as in Podinovski et al. (2016).

Proof of Proposition 4. If both sets $A$ and $B$ have a single element, Assumption 5 is identical to Assumption 4, and Proposition 4 is a restatement of Proposition 3.

Proof of Proposition 5. A pair $\left(\gamma^{\prime}, \varphi^{\prime}\right)$ is feasible in program (2) if and only if the pair $\left(\gamma^{\prime}, \varphi^{\prime}-\bar{\varphi}\left(\gamma^{\prime}\right)\right)$ is feasible in a similar program $(2)$ restated for the projected unit $\left(X^{*}, V^{*}, W^{*}\right)$. Therefore, $\Gamma^{*}=\Gamma$.

Consider program (C.1) restated for the function $\bar{\varphi}^{*}(\gamma)$. This requires changing the vectors $X_{o}^{B}, V_{o}^{B}$ and $W_{o}^{B}$ to, respectively, the vectors $X_{o}^{B}+\bar{\varphi}(0) \Delta_{X}^{B}, V_{o}^{B}+\bar{\varphi}(0) \Delta_{V}^{B}$ and $W_{o}^{B}+$ $\bar{\varphi}(0) \Delta_{W}^{B}$ on the right-hand side of the constraints (C.1c), (C.1f), (C.1i) and (C.1m). Note that the right-hand side of constraint (C.1f) becomes $-V_{o}^{B}-\bar{\varphi}(0) \Delta_{V}^{B}$. The objective function of the dual program (C.2), and the left-hand side of constraint (B.1c) of program (B.1) based on it, change accordingly and include the additional term

$$
\bar{\varphi}(0)\left[\left(\Delta_{X}^{B}\right)^{\top} v^{B}-\left(\Delta_{V}^{B}\right)^{\top} u^{B}+\left(\Delta_{W}^{B}\right)^{\top} z^{B}+\left(\Delta_{V}^{B}\right)^{\top} \zeta\right] .
$$

Taking into account constraint (B.1b), the above term is equal to $-\bar{\varphi}(0)$. Therefore, constraint (B.1c) is restated by changing its right-hand side from 0 to $\bar{\varphi}(0)$. 


\section{References}

Atici, K. B., \& Podinovski, V. V. (2012). Mixed partial elasticities in constant returns-to-scale production technologies. European Journal of Operational Research, 220(1), 262-269.

Asmild, M., Paradi, J. C., \& Reese, D. N. (2006). Theoretical perspectives of trade-off analysis using DEA. Omega, $34(4), 337-343$.

Balk, B. M., Färe, R., \& Karagiannis, G. (2015). On directional scale elasticities. Journal of Productivity Analysis, 43(1), 99-104.

Banker, R. D., Charnes, A., \& Cooper, W. W. (1984). Some models for estimating technical and scale efficiencies in data envelopment analysis. Management Science, 30(9), 1078-1092.

Banker, R. D., Cooper, W. W., Seiford, L. M., \& Zhu, J. (2011). Returns to scale in DEA. In W. W. Cooper, L. M, Seiford, \& J. Zhu (Eds.), Handbook on data envelopment analysis. (2nd ed.). (pp. 41-70). New York: Springer Science + Busines Media.

Chambers, R. G., Chung, Y., \& Färe, R. (1996). Benefit and distance functions. Journal of Economic Theory, 70(2), 407-419.

Chambers, R. G., Chung, Y., \& Färe, R. (1998). Profit, directional distance functions, and Nerlovian efficiency. Journal of Optimization Theory and Applications, 98(2), 351-364.

Chambers, R. G., \& Färe, R. (2008). A "calculus" for data envelopment analysis. Journal of Productivity Analysis, 30(3), 169-175.

Chambers, R. G., Serra, T., \& Oude Lansink, A. (2014). On the pricing of undesirable state-contingent outputs. European Review of Agricultural Economics, 41(3), 485-509.

Charnes, A., Cooper, W. W., \& Rhodes, E. (1978). Measuring the efficiency of decision making units. European Journal of Operational Research, 2(6), 429-444.

Chen, C.-M., \& Delmas, M. A. (2012). Measuring eco-efficiency: A new frontier approach. Operations Research, 60(5), 1064-1079.

Cherchye, L., De Rock, B., Dierynck, B., Roodhooft, F., \& Sabbe, J. (2013). Opening the "black box" of efficiency measurement: Input allocation in multioutput settings. Operations Research, 61(5), 1148-1165.

Dakpo, K. H., Jeanneaux, P., \& Latruffe, L. (2016). Modelling pollution-generating technologies in performance benchmarking: Recent developments, limits and future prospects in the nonparametric framework. European Journal of Operational Research, 250(2), 347-359.

Färe, R., Grosskopf, S., Lovell, C. A. K., \& Pasurka, C. (1989). Multilateral productivity comparisons when some outputs are undesirable: A nonparametric approach. The Review of Economics and Statistics, $71(1), 90-98$.

Färe, R., Grosskopf, S., Lovell, C. A. K., \& Yaisawarng, S. (1993). Derivation of shadow prices for undesirable outputs: A distance function approach. The Review of Economics and Statistics, 75 (2), 374-380.

Färe, R., Grosskopf, S., Noh, D.-W., \& Weber, W. (2005). Characteristics of a polluting technology: Theory and practice. Journal of Econometrics, 126(2), 469-492.

Førsund, F. R. (2018). Economic interpretations of DEA. Socio-Economic Planning Sciences, 61, 9-15.

Førsund, F. R. (1996). On the calculation of scale elasticity in DEA models. Journal of Productivity Analysis, $7(2-3), 283-302$.

Førsund, F. R., \& Hjalmarsson, L. (2004). Calculating scale elasticity in DEA models. Journal of the Operational Research Society, 55(10), 1023-1038.

Frisch, R. (1965). Theory of production. Dordrecht: D. Reidel Publishing Company.

Fukuyama, H., \& Matousek, R. (2018). Nerlovian revenue inefficiency in a bank production context: Evidence from Shinkin banks. European Journal of Operational Research, 271(1), 317-330.

Kao, C. (2014). Network data envelopment analysis: A review. European Journal of Operational Research, $239(1), 1-16$.

Kuosmanen, T. (2005). Weak disposability in nonparametric productivity analysis with undesirable outputs. American Journal of Agricultural Economics, 87(4), 1077-1082.

Kuosmanen, T., \& Kazemi Matin, R. (2011). Duality of weakly disposable technology. Omega, 39(5), 504512 .

Kuosmanen, T., \& Podinovski, V. V. (2009). Weak disposability in nonparametric production analysis: Reply to Färe and Grosskopf. American Journal of Agricultural Economics, 91(2), 539-545.

Lee, S.-C., Oh, D.-H., \& Lee, J.-D. (2014). A new approach to measuring shadow price: Reconciling engineering and economic perspectives. Energy Economics, 46, 66-77.

Lee, J.-D., Park, J.-B., \& Kim, T.-Y. (2002). Estimation of the shadow prices of pollutants with production/environment inefficiency taken into account: A nonparametric directional distance function approach. Journal of Environmental Management, 64 (4), 365-375. 
Lee, C.-Y., \& Zhou, P. (2015). Directional shadow price estimation of $\mathrm{CO}_{2}, \mathrm{SO}_{2}$ and $\mathrm{NO}_{\mathrm{x}}$ in the United States coal power industry 1990-2010. Energy Economics, 51, 493-502.

Mehdiloo, M., \& Podinovski, V. V. (2019). Selective strong and weak disposability in efficiency analysis. European Journal of Operational Research, 276(3), 1154-1169.

Mehdiloozad, M., \& Podinovski, V. V. (2018). Nonparametric production technologies with weakly disposable inputs. European Journal of Operational Research, 266(1), 247-258.

Mekaroonreung, M., \& Johnson, A. L. (2012). Estimating the shadow prices of $\mathrm{SO}_{2}$ and $\mathrm{NO}_{\mathrm{x}}$ for U.S. coal power plants: A convex nonparametric least squares approach. Energy Economics, 34(3), 723-732.

Pham, M. D., \& Zelenyuk, V. (2019). Weak disposability in nonparametric production analysis: A new taxonomy of reference technology sets. European Journal of Operational Research, 274(1), 186-198.

Podinovski, V. V. (2004a). Bridging the gap between the constant and variable returns-to-scale models: Selective proportionality in data envelopment analysis. Journal of the Operational Research Society, 55 (3), 265-276.

Podinovski, V. V. (2004b). Production trade-offs and weight restrictions in data envelopment analysis. Journal of the Operational Research Society, 55(12), 1311-1322.

Podinovski, V. V. (2017). Returns to scale in convex production technologies. European Journal of Operational Research, 258(3), 970-982.

Podinovski, V. V., \& Bouzdine-Chameeva, T. (2013). Weight restrictions and free production in data envelopment analysis. Operations Research, 61(2), 426-437.

Podinovski, V. V., Chambers, R. G., Atici, K. B., \& Deineko, I. D. (2016). Marginal values and returns to scale for nonparametric production frontiers. Operations Research, 64 (1), 236-250.

Podinovski, V. V., \& Førsund, F. R. (2010). Differential characteristics of efficient frontiers in data envelopment analysis. Operations Research, 58(6), 1743-1754.

Podinovski, V. V., Førsund, F. R., \& Krivonozhko, V. E. (2009). A simple derivation of scale elasticity in data envelopment analysis. European Journal of Operational Research, 197(1), 149-153.

Podinovski, V. V., \& Kuosmanen, T. (2011). Modelling weak disposability in data envelopment analysis under relaxed convexity assumptions. European Journal of Operational Research, 211(3), 577-585.

Podinovski, V. V., Olesen, O. B., \& Sarrico, C. S. (2018). Nonparametric production technologies with multiple component processes. Operations Research, 66(1), 282-300.

Sahoo, B. K., \& Tone, K. (2015). Scale elasticity in non-parametric DEA approach. In J. Zhu (Ed.), Data envelopment analysis: A handbook of models and methods (pp. 269-290). New York: Springer Science + Busines Media.

Starrett, D. A. (1977). Measuring returns to scale in the aggregate, and the scale effect of public goods. Econometrica, 45(6), 1439-1455.

Zhou, P., Zhou, X., \& Fan, L. W. (2014). On estimating shadow prices of undesirable outputs with efficiency models: A literature review. Applied Energy, 130, 799-806. 
Table 5: The data set in the computational example.

\begin{tabular}{|c|c|c|c|c|c|}
\hline Unit & Input $X_{1}$ & Input $X_{2}$ & Good output $V_{1}$ & Bad output $W_{1}$ & Bad output $W_{2}$ \\
\hline 1 & 27.88 & 4.24 & 8.38 & 2.5 & 0.82 \\
\hline 2 & 23.61 & 13.35 & 11.03 & 7.79 & 3.27 \\
\hline 3 & 24.23 & 17.01 & 15.07 & 10.05 & 3.85 \\
\hline 4 & 17.97 & 1.93 & 4.29 & 1.1 & 0.5 \\
\hline 5 & 35.94 & 6.79 & 9.87 & 3.95 & 1.68 \\
\hline 6 & 45.43 & 12.85 & 18.31 & 7.65 & 2.12 \\
\hline 7 & 40.58 & 14.26 & 15.3 & 7.38 & 3.22 \\
\hline 8 & 45.84 & 8.87 & 13.03 & 4.67 & 1.31 \\
\hline 9 & 31.45 & 4.59 & 7.63 & 2.37 & 1.11 \\
\hline 10 & 46.05 & 42.18 & 29.45 & 24.66 & 4.41 \\
\hline 11 & 23.21 & 12.94 & 13.33 & 6.98 & 3.85 \\
\hline 12 & 31.65 & 10.62 & 12.8 & 6.37 & 2.51 \\
\hline 13 & 34.83 & 36.72 & 21.18 & 19.75 & 4.34 \\
\hline 14 & 14.05 & 6.32 & 6.14 & 3.76 & 1.53 \\
\hline 15 & 31.51 & 20.58 & 14.65 & 11.43 & 5.21 \\
\hline 16 & 17.9 & 5.87 & 7.05 & 3.5 & 1 \\
\hline 17 & 17.61 & 11.73 & 10.86 & 5.93 & 1.98 \\
\hline 18 & 12.29 & 3.13 & 4.66 & 1.84 & 0.47 \\
\hline 19 & 31.58 & 19.62 & 14.58 & 11.47 & 2.01 \\
\hline 20 & 33.03 & 17.72 & 14.72 & 10.25 & 2.95 \\
\hline 21 & 26.55 & 12.7 & 13.22 & 6.71 & 2.74 \\
\hline 22 & 30.85 & 20.54 & 15.23 & 11.26 & 3.91 \\
\hline 23 & 31.35 & 18.37 & 13.3 & 9.97 & 3.93 \\
\hline 24 & 20.36 & 7.37 & 9.09 & 3.8 & 1.07 \\
\hline 25 & 43.82 & 8.09 & 13.58 & 4.31 & 0.86 \\
\hline 26 & 17.61 & 13.02 & 9.2 & 7.54 & 2.61 \\
\hline 27 & 49.63 & 52.3 & 28.04 & 30.58 & 5.79 \\
\hline 28 & 44.24 & 44.27 & 30.19 & 24.5 & 4.44 \\
\hline 29 & 35.21 & 25.45 & 20.19 & 13.39 & 3.64 \\
\hline 30 & 22.81 & 2.66 & 6.87 & 1.54 & 0.29 \\
\hline 31 & 16.93 & 8.18 & 7.93 & 4.72 & 1.5 \\
\hline 32 & 26.43 & 10.48 & 11.86 & 5.94 & 1.11 \\
\hline 33 & 45.4 & 42.76 & 23.76 & 22.62 & 4.95 \\
\hline 34 & 39.91 & 8.72 & 14.6 & 5 & 1.68 \\
\hline 35 & 21.33 & 5.65 & 6.79 & 2.92 & 0.96 \\
\hline 36 & 33.57 & 12.55 & 11.27 & 6.35 & 2.04 \\
\hline 37 & 39.81 & 12.08 & 13.73 & 6.99 & 2.05 \\
\hline 38 & 47.55 & 44.06 & 24.18 & 25.78 & 7.58 \\
\hline 39 & 46.82 & 15.47 & 17.77 & 7.85 & 3.29 \\
\hline 40 & 34.83 & 27.25 & 19.69 & 16.24 & 6.87 \\
\hline 41 & 23.63 & 8.77 & 8.24 & 4.6 & 1.65 \\
\hline 42 & 15.21 & 3.01 & 5.28 & 1.75 & 0.83 \\
\hline 43 & 47.35 & 13.82 & 18.7 & 7.98 & 3.62 \\
\hline 44 & 13.53 & 3.55 & 5.33 & 1.8 & 0.49 \\
\hline 45 & 33.86 & 37.1 & 19.89 & 20.61 & 7.56 \\
\hline
\end{tabular}


Table 6: Results of computations.

\begin{tabular}{|c|c|c|c|c|c|c|c|}
\hline \multirow[b]{2}{*}{ Unit } & \multicolumn{3}{|c|}{ Direct estimation } & \multicolumn{4}{|c|}{ Estimation based on shadow prices } \\
\hline & $\bar{\varphi}(0)$ & $\partial^{+} V_{1} / \partial W_{1}$ & $\partial^{-} V_{1} / \partial W_{1}$ & $\vec{D}(\cdot)$ & $\partial \vec{D}(.) / \partial V_{1}$ & $\partial \vec{D}\left(\dot{)} / \partial W_{1}\right.$ & $-\frac{\partial \vec{D}(\cdot) / \partial W_{1}}{\partial \vec{D}(\cdot) / \partial V_{1}}$ \\
\hline 1 & 0 & -253.231 & 1.2344 & 0.1642 & -0.5295 & 1.6614 & 3.1376 \\
\hline 2 & 1.7063 & -5.798 & -5.798 & 1.3721 & -0.3674 & 0.2062 & 0.5613 \\
\hline 3 & 0 & -43.763 & 0.4114 & 0 & -0.9086 & 0.3738 & 0.4114 \\
\hline 4 & 0 & $-\infty$ & $+\infty$ & 0 & -0.2995 & 0.8897 & 2.9708 \\
\hline 5 & 0.6108 & -16.582 & -16.582 & 0.7441 & -0.376 & 0.9853 & 2.6201 \\
\hline 6 & 0 & $-\infty$ & 1.4083 & 0 & -0.2345 & 0.0695 & 0.2963 \\
\hline 7 & 1.6464 & 1.0658 & 1.0658 & 0.8825 & -0.536 & 0.5713 & 1.0658 \\
\hline 8 & 1.0609 & 2.6201 & 2.6201 & 0.3989 & -0.376 & 0.9853 & 2.6201 \\
\hline 9 & 0 & 0.9721 & 8.4987 & 0 & -0.5295 & 1.6614 & 3.1376 \\
\hline 10 & 0 & $-\infty$ & 0.0667 & 0 & 0 & -0.2224 & Undefined \\
\hline 11 & 0 & $-\infty$ & $+\infty$ & 0 & -0.4671 & 0.545 & 1.1669 \\
\hline 12 & 0 & $-\infty$ & -3.5815 & 0.7032 & -0.4133 & 0.3588 & 0.8682 \\
\hline 13 & 0 & -9.7507 & -1.5098 & 0 & -0.2586 & -1.3787 & -5.3311 \\
\hline 14 & 0 & $-\infty$ & $+\infty$ & 0.5584 & -0.532 & -1.202 & -2.2594 \\
\hline 15 & 2.3168 & 0.778 & 0.778 & 2.0242 & -0.5129 & 0.351 & 0.6843 \\
\hline 16 & 0 & $-\infty$ & -13.8777 & 0.3197 & -0.1016 & -0.1001 & -0.9849 \\
\hline 17 & 0 & -2.2594 & 1.1506 & 0 & -0.324 & 0.1625 & 0.5014 \\
\hline 18 & 0 & $-\infty$ & $+\infty$ & 0 & -0.0427 & -0.1833 & -4.2976 \\
\hline 19 & 0 & $-\infty$ & -4.6644 & 0 & -0.0049 & -0.2039 & -41.492 \\
\hline 20 & 2.4661 & -0.3786 & -0.3786 & 1.1586 & -0.2539 & 0.092 & 0.3623 \\
\hline 21 & 0.3678 & 0.9365 & 0.9365 & 0.1747 & -0.4749 & 0.4448 & 0.9365 \\
\hline 22 & 2.7077 & 0.7386 & 0.7386 & 1.4143 & -0.5129 & 0.351 & 0.6843 \\
\hline 23 & 3.7509 & 0.778 & 0.778 & 1.9153 & -0.3674 & 0.2062 & 0.5613 \\
\hline 24 & 0 & -0.2704 & 1.1669 & 0 & -0.3006 & 0.1918 & 0.6382 \\
\hline 25 & 0 & -8.7843 & 2.6201 & 0 & -0.023 & -0.1831 & -7.9787 \\
\hline 26 & 0 & $-\infty$ & 0.7398 & 0 & -0.532 & -1.202 & -2.2594 \\
\hline 27 & 0 & $-\infty$ & 0.0785 & 0 & 0 & -0.3456 & Undefined \\
\hline 28 & 0 & $-\infty$ & 0.7786 & 0 & -0.0396 & -0.1847 & -4.6644 \\
\hline 29 & 0.1607 & 0.6843 & 0.6843 & 0.0824 & -0.5129 & 0.351 & 0.6843 \\
\hline 30 & 0 & $-\infty$ & 4.8579 & 0 & -0.0049 & -0.2039 & -41.492 \\
\hline 31 & 0.5354 & -0.909 & -0.909 & 0.425 & -0.3075 & 0.111 & 0.361 \\
\hline 32 & 0 & -5.2894 & 0.701 & 0 & -0.0499 & -0.1544 & -3.094 \\
\hline 33 & 4.1123 & 0.7786 & 0.7786 & 1.1139 & 0 & -0.2224 & Undefined \\
\hline 34 & 0 & -7.9044 & 3.2862 & 0 & -0.3117 & 0.2494 & 0.8003 \\
\hline 35 & 1.4807 & 1.1669 & 1.1669 & 0.4803 & -0.0427 & -0.1833 & -4.2976 \\
\hline 36 & 3.3298 & 0.9365 & 0.9365 & 1.0386 & -0.0499 & -0.1544 & -3.094 \\
\hline 37 & 2.7713 & 0.4102 & 0.4102 & 0.8139 & -0.2539 & 0.092 & 0.3623 \\
\hline 38 & 0 & $-\infty$ & $+\infty$ & 3.5841 & -0.0709 & -0.1294 & -1.8251 \\
\hline 39 & 0.7634 & 1.4083 & 1.4083 & 0.3237 & -0.424 & 0.5971 & 1.4083 \\
\hline 40 & 0 & $-\infty$ & $+\infty$ & 0 & -1.2465 & 0.9698 & 0.778 \\
\hline 41 & 2.4524 & 1.0729 & 1.0729 & 0.9198 & -0.3006 & 0.1918 & 0.6382 \\
\hline 42 & 0 & $-\infty$ & $+\infty$ & 0 & -0.5331 & 0.9256 & 1.7362 \\
\hline 43 & 0 & -61.7041 & 4.2167 & 0 & -0.5561 & 0.3838 & 0.6902 \\
\hline 44 & 0 & -0.9849 & 3.3258 & 0 & -0.1016 & -0.1001 & -0.9849 \\
\hline 45 & 0 & $-\infty$ & $+\infty$ & 0 & -3.7687 & 1.2651 & 0.3357 \\
\hline
\end{tabular}


Table B.7: The data set for Example 4.

\begin{tabular}{ccccc}
\hline Unit & Input $X$ & Good output $V_{1}$ & Good output $V_{2}$ & Bad output $W$ \\
\hline$A$ & 0.5 & 1.5 & 1 & 2 \\
$B$ & 1 & 1 & 0 & 1 \\
\hline
\end{tabular}

Table B.8: One-sided marginal rates $\partial^{-} W / \partial V_{2}$ and $\partial^{+} W / \partial V_{2}$ in Example 4.

\begin{tabular}{ccc}
\hline Unit & $\partial^{-} W / \partial V_{2}$ & $\partial^{+} W / \partial V_{2}$ \\
\hline$A$ & 0 & $+\infty$ \\
$B$ & $-\infty$ & 0.5 \\
\hline
\end{tabular}

Table B.9: One-sided derivatives $\bar{\varphi}_{-}^{\prime}(0)$ and $\bar{\varphi}_{+}^{\prime}(0)$ in Example 5.

\begin{tabular}{ccc}
\hline Unit & $\bar{\varphi}_{-}^{\prime}(0)$ & $\bar{\varphi}_{+}^{\prime}(0)$ \\
\hline$A$ & 0.75 & $-\infty$ \\
$B$ & $+\infty$ & 0.5 \\
\hline
\end{tabular}

